\title{
Multi-modality image-based computational analysis of haemodynamics in aortic dissection
}

\author{
Desmond Dillon-Murphy ${ }^{1}$ - Alia Noorani ${ }^{1}$ - David Nordsletten ${ }^{1}$. \\ C. Alberto Figueroa ${ }^{1,2}$
}

Received: 20 May 2015 / Accepted: 10 September 2015 / Published online: 28 September 2015

(C) The Author(s) 2015. This article is published with open access at Springerlink.com

\begin{abstract}
Aortic dissection is a disease whereby an injury in the wall of the aorta leads to the creation of a true lumen and a false lumen separated by an intimal flap which may contain multiple communicating tears between the lumina. It has a high associated morbidity and mortality, but at present, the timing of surgical intervention for stable type B dissections remains an area of debate. Detailed knowledge of haemodynamics may yield greater insight into the long-term outcomes for dissection patients by providing a greater understanding of pressures, wall shear stress and velocities in and around the dissection. In this paper, we aim to gather further insight into the complex haemodynamics in aortic dissection using medical imaging and computational fluid dynamics modelling. Towards this end, several computer models of the aorta of a patient presenting with an acute Stanford type B dissection were created whereby morphometric parameters related to the dissection septum were altered, such as removal of the septum, and the variation of the number of connecting tears between the lumina. Patient-specific flow data acquired using 2D PC-MRI in the ascending aorta were used to set the inflow boundary condition. Coupled zero-dimensional (Windkessel) models representing the distal vasculature were used to define the outlet boundary conditions and tuned to match 2D PC-MRI flow data acquired in the descending aorta. Haemodynamics in the dissected aorta were compared to those in an equivalent 'healthy aorta', created by virtually removing the intimal flap (septum). Local regions of
\end{abstract}

$\triangle$ C. Alberto Figueroa

figueroc@med.umich.edu

1 Department of Biomedical Engineering, King's College London, London SE1 7EH, UK

2 Departments of Surgery and Biomedical Engineering, University of Michigan, North Campus Research Complex B20-211W, Ann Arbor, MI 48109, USA increased velocity, pressure, wall shear stress and alterations in flow distribution were noted, particularly in the narrow true lumen and around the primary entry tear. The computed flow patterns compared favourably with those obtained using 4D PC-MRI. A lumped-parameter heart model was subsequently used to show that in this case there was an estimated $14 \%$ increase in left ventricular stroke work with the onset of dissection. Finally, the effect of secondary connecting tears (i.e. those excluding the primary entry and exit tears) was also studied, revealing significant haemodynamic changes when no secondary tears are included in the model, particularly in the true lumen where increases in flow over $+200 \%$ and drops in peak pressure of $18 \%$ were observed.

Keywords CFD · Aortic dissection .

Multi-scale modelling · Cardiac work load · Intimal tears

\section{Contents}

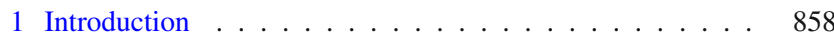

2 Material and methods . . . . . . . . . . . . . . . . . . . . . . . . . . . . 859

2.1 Patients details . . . . . . . . . . . . . . . . . . . . . . . . . 859

2.2 Imaging data . . . . . . . . . . . . . . . . . . . . . 859

2.2.1 Anatomy: CT data . . . . . . . . . . . . . . . . . 859

2.2.2 Flow data: MRI data . . . . . . . . . . . . . . . . . 859

2.3 Geometric modelling . . . . . . . . . . . . . . . 861

2.4 Computational fluid dynamics . . . . . . . . . 862

2.4.1 Coupled multi-domain method for blood flow simu-

lation . . . . . . . . . . . 862

2.4.2 Mesh generation and time integration strategy . . 863

3 Results . . . . . . . . . . . . . . . . . . . 864

3.1 Study 1: Haemodynamic alterations in aortic dissection 864

3.1.1 Baseline haemodynamics in undissected aorta . . 864

3.1.2 Dissected aorta . . . . . . . . . . . . . . . 864

3.1.3 Comparison between computed and measured flows

in the descending aorta . . . . . . . . 866

3.2 Study 2: Analysis of the impact of the flap on left ventricular function .................. 867 
3.3 Study 3: Sensitivity study of the impact of secondary entry tears . . . . . . . . . . . . . 868

4 Discussion . . . . . . . . . . . . . . . . 870

4.1 Limitations and future work . . . . . . . . . . . 872

4.1 .1 Imaging limitations . . . . . . . . . . . . . . . . . . . . . 872

4.1 .2 Pressure data . . . . . . . . . . . . . . . . 873

4.1.3 Modelling limitations . . . . . . . . . . . . . . . 873

5 Conclusions . . . . . . . . . . . . . . . . . . 874

References . . . . . . . . . . . . . . . . . 874

\section{Introduction}

In aortic dissection, a tear in the aortic intima creates a true and false lumen for blood flow. Regardless of the type of dissection (Stanford type A or B), patients have a poor long-term prognosis with $50 \%$ mortality at 5 years (Tsai et al. 2007). The majority of deaths occur due to false lumen expansion, further dissection and rupture. Current anatomical predictors of adverse outcomes include initial aortic size, a patent (or partially thrombosed false lumen) and Marfan's syndrome. Such anatomical predictors of poor outcomes are used to customise follow-up strategies and optimise treatment planning. However, physiological indices such as intra-aortic haemodynamics (pressure and flow) may, in fact, provide improved patient-specific predictors of outcomes.

A number of studies (Karmonik et al. 2008; Rudenick et al. 2010; Karmonik et al. 2010; Cheng et al. 2010; Karmonik et al. 2011b; Tse et al. 2011; Karmonik et al. 2011a, 2012; Chen et al. 2013; Karmonik et al. 2013; Alimohammadi et al. 2014) have shown that analysis of blood flow through patientspecific geometries using computational fluid dynamics can yield significant insight into the complex haemodynamics present in dissection patients which may be difficult or impossible to quantify with other means. For example, in a longitudinal study involving a dissection patient with image data acquired pre- and post-aneurysmal development Tse et al. (2011) investigated the influence that the aneurysm has on haemodynamics. A similar study can be found in Karmonik et al. (2012). Karmonik also investigated how flow and pressure patterns in the vicinity of the dissection are affected when either the entry tear or exit tear is occluded (Karmonik et al. 2010), haemodynamic changes pre- and post-EVAR (Karmonik et al. 2011b), as well as a comparison between haemodynamics in a healthy subject and those in a patient with dissection (Karmonik et al. 2013). Rudenick et al. (2013) performed a combined experimental and computational study using an idealised rig of a dissection, comparing measured and simulated flows in both the true and false lumina, showing good agreement. In Chen et al. (2013), the haemodynamics of a dissection patient were studied in a model that included primary entry and exit tears, as well as a secondary tear. Lastly, Alimohammadi et al. (2014) per- formed a study in a simpler dissection model using coupled RCR Windkessel models at the outlet branches.

Secondary intimal tears in the dissection flap are thought to occur due to avulsion around branching vessels as the dissection propagates (Willoteaux et al. 2004). Studies have found that patients with dissection generally present with more than two tears (Quint et al. 2003; Khoynezhad et al. 2010). However, as it has been shown, tears may be difficult to identify in the medical image data, due to the small size and potential motion artefacts in the septum. In this study, we used a combination of two imaging modalities, CTA and 4D PC-MRI, to try to detect the location of these secondary tears.

In this paper, the haemodynamic impact of different aortic dissection morphometric parameters is investigated by conducting the following three novel studies:

1. An analysis of the haemodynamic changes introduced by the dissection septum in the descending aorta. Here, a comparison of haemodynamic predictions was made between a multi-branched, multi-scale model of aortic dissection and a baseline 'healthy' aortic model created by virtually removing the septum. This approach enables us to investigate specific changes in blood flow, pressure, wall shear stress, etc. directly attributable to the dissection.

2. An estimation of the additional stroke work imposed by the aortic dissection on the heart using a tuned lumpedparameter heart model.

3. A quantification of the impact of the often ignored secondary tears on aortic haemodynamics. These tears, typically found in the vicinity of visceral branches, are difficult to visualise due to limitations in the image resolution and motion artefacts. In this study, three different dissection models with varying number of tears were investigated. The results demonstrate that while these tears may be difficult to image, they can, if present, have a significant influence on the haemodynamics.

In all previous studies on aortic haemodynamics, a validation of numerical predictions against in vivo imaging data is lacking. We aim to address this and validate our results by using rich medical imaging data (computed tomography, 2D PC-MRI and 4D MRI) to assess the quality of our computer simulations, comparing simulated and measured flows in several true lumen and false lumen locations in the descending aorta of a patient with an acute type B dissection.

The structure of this paper is as follows: in Sect. 2, details on patient and imaging data, geometric modelling approach and computational tools are provided. In Sect. 3, the above-mentioned studies are presented. Lastly, the paper is concluded by discussing a set of limitations on both the imaging and the modelling sides that must be overcome to 


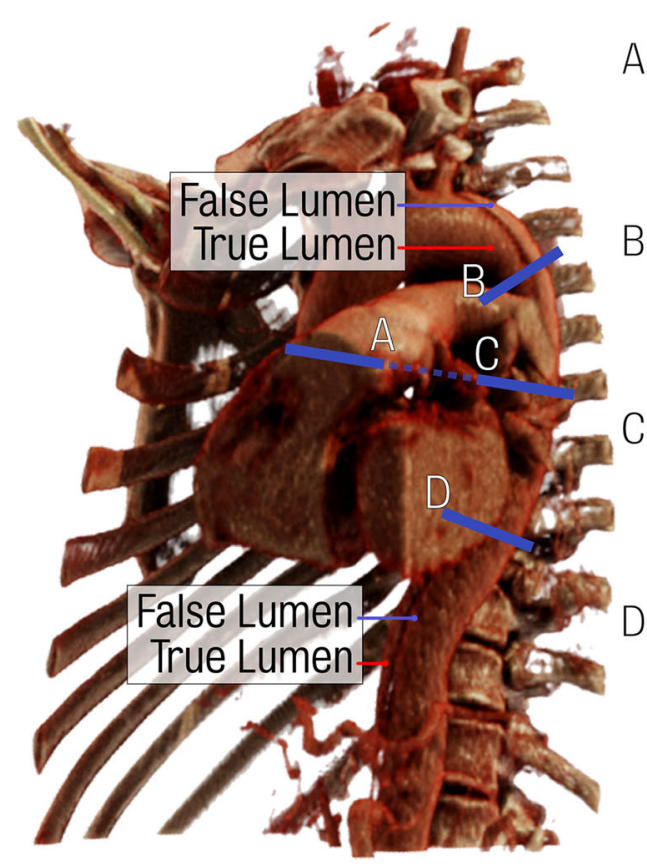

CT Volume Render Thoracic Aorta

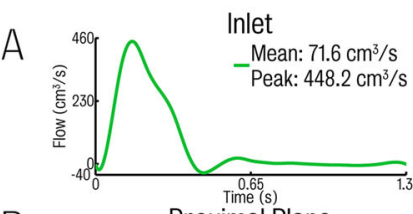

B

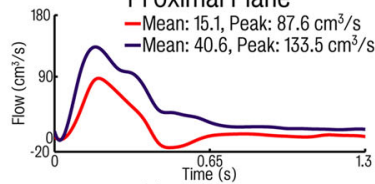

Medial Plane

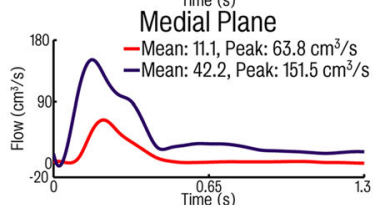

Distal Plane

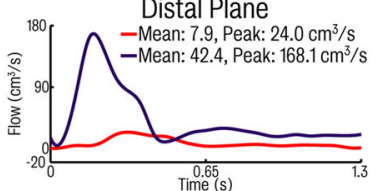

-True Lumen -False Lumen

2D PC-MRI

Flow Quantification

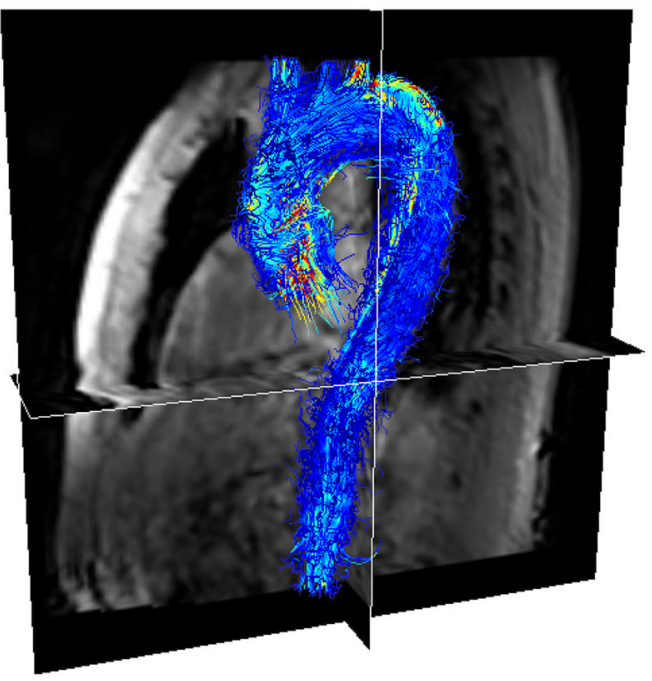

4D PC-MRI

Flow Quantification

Fig. 1 Left CT volume render of the thoracic aorta illustrating the dissection septum. Centre the 2D PC-MRI data at aortic inflow and several locations down the aorta. Right full aorta 4D PC-MRI data

enhance the accuracy of image-based modelling of aortic dissection.

\section{Material and methods}

\subsection{Patients details}

A 49-year-old male subject presented with an acute type B dissection originating $5 \mathrm{~mm}$ distal of the left subclavian artery, extending the full length of the descending aorta and terminating $5 \mathrm{~mm}$ distal to the left iliac bifurcation. The long axis of the dissection septum progressively rotates approximately $90^{\circ}$ clockwise along the length of the descending thoracic aorta, bisecting it largely in the sagittal direction in the abdominal region. A number of vessels branch from the false lumen (left renal artery, inferior mesenteric artery and medial sacral artery), while the remaining branch from the true lumen. It should also be noted that several vessels in the coeliac trunk region (coeliac trunk, superior mesenteric artery) have, due to their proximity of the dissection flap, a significantly "pinched" appearance at the branching location from the aorta, resulting in an oval cross section with the short axis aligned with the transverse plane.

The patient was previously prescribed antihypertensive drugs (Amlodipine $10 \mathrm{mg}$, Candesartan $4 \mathrm{mg}$ ) to treat a his- tory of high blood pressure pre the dissection event. On presentation, the patient was administered additional intravenous antihypertensive medication to acutely control blood pressure.

\subsection{Imaging data}

\subsubsection{Anatomy: CT data}

A 40-row multi-slice scanner (Brilliance 40; Philips Medical Systems, Cleveland, Ohio) was used to image the full aorta in two separate scans acquired using first-pass bolus-tracked contrast-enhanced images during one breath hold: one capturing the thoracic aorta and the second the abdominal aorta (see Figs. 1, 2); $100 \mathrm{~mL}$ of iohexol contrast agent (Omnipaque 350; GE Healthcare, Oslo, Norway) was delivered at $4 \mathrm{~mL} / \mathrm{s}$ using a power injector (Medrad Spectris, Siemens Medical Solutions, Malvern, Pa). The scan was automatically triggered when the contrast enhancement reached 150 HU. Settings for both the thoracic and abdominal scans are listed in Table 1.

\subsubsection{Flow data: MRI data}

In this work, 2D PC-MRI data were used to quantify blood flow at four different locations, while 4D PC-MRI data were 


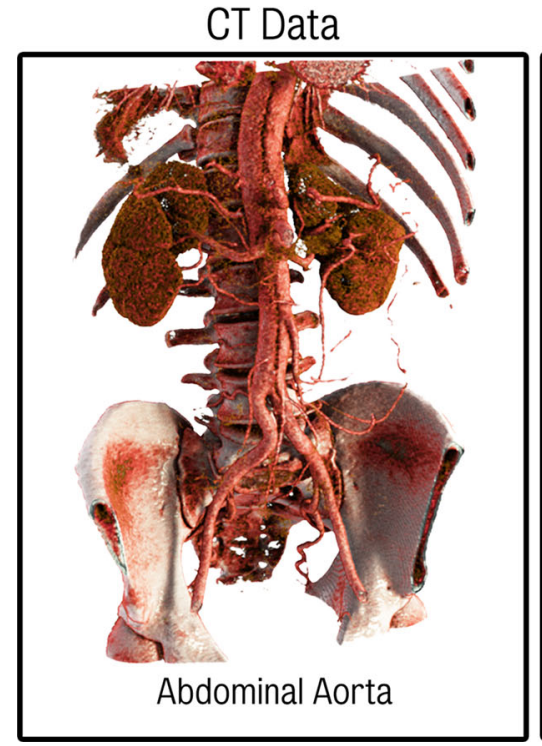

Fig. 2 Left CTA showing the anatomy of the dissection in the abdominal aorta. The small size and motion of the septum make it difficult to clearly delineate the true and false lumen. Right 3D solid model creation process using a 2D segmentation approach. In step 1, contours of the lumina are created. These contours are then lofted together (step 2) to create a non-uniform rational B-spline (NURBS) analytical representa-

Table 1 Scan settings for both thoracic and abdominal CT scans of an aortic dissection subject

\begin{tabular}{lll}
\hline Property & Thoracic & Abdominal \\
\hline FOV & $376 \mathrm{~mm}$ & $280 \mathrm{~mm}$ \\
In plane resolution & $512 \times 512$ & $512 \times 512$ \\
Slice thickness & $2 \mathrm{~mm}$ & $0.9 \mathrm{~mm}$ \\
Tilt & $0^{\circ}$ & $0^{\circ}$ \\
Tube voltage (KPV) & $120 \mathrm{kV}$ & $120 \mathrm{kV}$ \\
Tube current & $412 \mathrm{~mA}$ & $221 \mathrm{~mA}$ \\
Acquisition & Cephalocaudal & Cephalocaudal \\
\hline
\end{tabular}

used to perform a qualitative comparison between imaging and CFD-derived velocity fields. All image data were collected using the methods presented in Clough et al. (2012) and are summarised here for completeness.

A thirty-two element coil Achieva 3T scanner (Philips Healthcare, Best, the Netherlands) was used to acquire the images. Image reconstruction was performed using a Philips scanner console and commercially available software. Eddy currents and Maxwell phase offsets in the 2D PC-MRI and 4D PC-MRI data were corrected for using standard Philips software algorithms (Bernstein et al. 1998; Markl et al. 2003; Gatehouse et al. 2010).

Flow-encoded through-pane images were acquired at three positions perpendicular to the longitudinal axis of the aorta capturing flow. These locations included the ascending
2D Segmentation Process

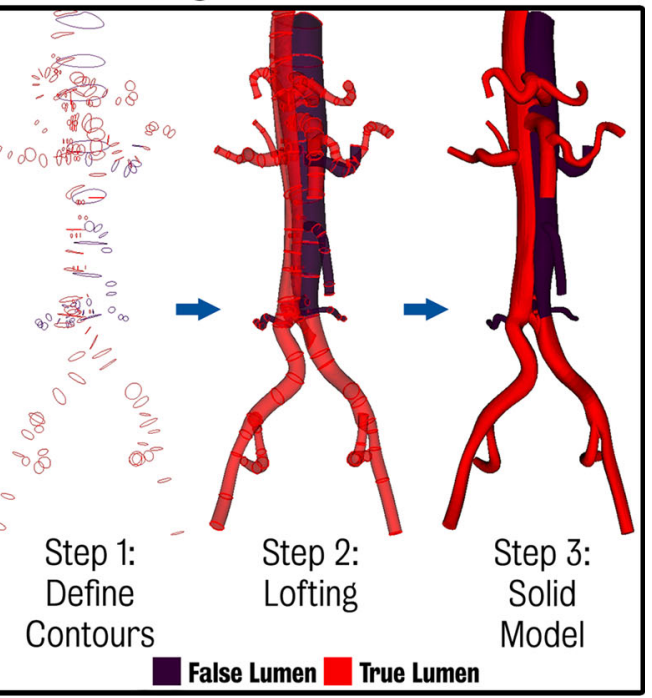

tion of each vessel. Finally, (step 3) the lofted surfaces are joined and blending operations using fixed radius fillets are employed to remove sharp edges between branching vessels. The false lumen and vessels perfused from the false lumen are coloured in purple, while all other vessels are coloured in red

Table 2 2D PC-MRI scan properties

\begin{tabular}{ll}
\hline Property & Value \\
\hline Field of View (FOV) & $350 \times 350 \mathrm{~mm}^{2}$ \\
Voxel size & $1.74 \times 1.99 \mathrm{~mm}^{2}$ \\
Slice thickness & $10 \mathrm{~mm}$ \\
Velocity encoding & $150 \mathrm{~cm} / \mathrm{s}$ \\
TR/TE & $5.0 / 3.0 \mathrm{~ms}$ \\
Temporal resolution & $52 \mathrm{~ms}$ \\
Number of signal averages & 2 \\
\hline
\end{tabular}

aorta just distal to the aortic valve (this plane also acquired flow in the descending aorta at the level of the T-4 vertebra), the descending aorta, $15 \mathrm{~mm}$ distal to the left subclavian and the descending aorta at the level of the T-7 vertebra. The position of these planes is noted in blue in Fig 1. ECG-gated data were later allotted to 25 cardiac phases (details given in Table 2).

The reconstructed images were checked for phase wraps immediately after acquisition. If present, velocity encoding was increased to ensure that encoding for the peak velocity was attained. Stroke volume and heart rate were found to be $94 \mathrm{~mL}$ and heart rate $47 \mathrm{BPM}$, respectively. This corresponds to a cardiac output of $4.4 \mathrm{~L} / \mathrm{min}$. Velocity data were processed using the multi-dimensional MRI phase-contrast flow visualisation program, GTFlow (GyroTools LLC). 
Table 3 4D PC-MRI scan properties

\begin{tabular}{ll}
\hline Property & Value \\
\hline Field of view (FOV) & $320 \times 320 \times 85 \mathrm{~mm}^{3}$ \\
Voxel size & $2.0 \times 2.04 \times 8 \mathrm{~mm}^{3}$ \\
Flip angle & $45^{\circ}$ \\
Velocity encoding & $150 \mathrm{~cm} / \mathrm{s}$ \\
TR/TE & $3.0 / 2.0 \mathrm{~ms}$ \\
Temporal resolution & $52 \mathrm{~ms}$ \\
\hline
\end{tabular}

The entire thoracic aorta was covered using an oblique sagittal slab 4D MRI anatomy scan (see Fig. 1). ECG-gating and respiratory navigator were used for data acquisition of a single respiratory phase $(5-\mathrm{mm}$ gating window at end expiration). ECG-gated data were later allotted to 25 cardiac phases. A flow-sensitive, gradient and radio frequency-spoiled gradient echo (phase-contrast) sequence was used (details given in Table 3).

\subsection{Geometric modelling}

In order to create a geometric CAD model, the 2D segmentation paradigm originally introduced in Wang et al. (1999) was utilised. Using this approach, paths were defined through roughly the centreline of the vessels to be included in the model. Then, a 2D automatic segmentation operation along the paths was performed at a number of discrete locations. This 2D segmentation step provides the contours of true and false lumina of the dissection. Lastly, lofted surfaces are obtained using non-uniform rational B-splines (NURBS) to produce an analytical and smooth solid model that must then be meshed. The right panel of Fig. 2 depicts the various steps of this segmentation approach. The false lumen and vessels perfused from the false lumen are coloured in purple, while all other vessels are coloured in red.

Several models were created using this methodology. A baseline "dissected model" containing the primary entry and exit tears as well as 15 secondary connecting tears were generated from the CT data. Most of these secondary tears were located in the visceral region. These tears generally form in regions where the septum separates from the wall in the vicinity of a branching vessel, and are believed to be the remnants of the original entry to the branching vessel (Willoteaux et al. 2004). A large number of connecting tears may be observed near the coeliac trunk, renal artery, etc. (Tam et al. 1998; McMahon and Squirrell 2010; Takami et al. 2012). The size of the main entry and exit tears was 135 and $68 \mathrm{~mm}^{2}$, respectively. This corresponds to effective diameters of 13 and $9.3 \mathrm{~mm}$, respectively. From this baseline "dissected model", an "undissected model" was created by virtually removing the septum from the dissected model and

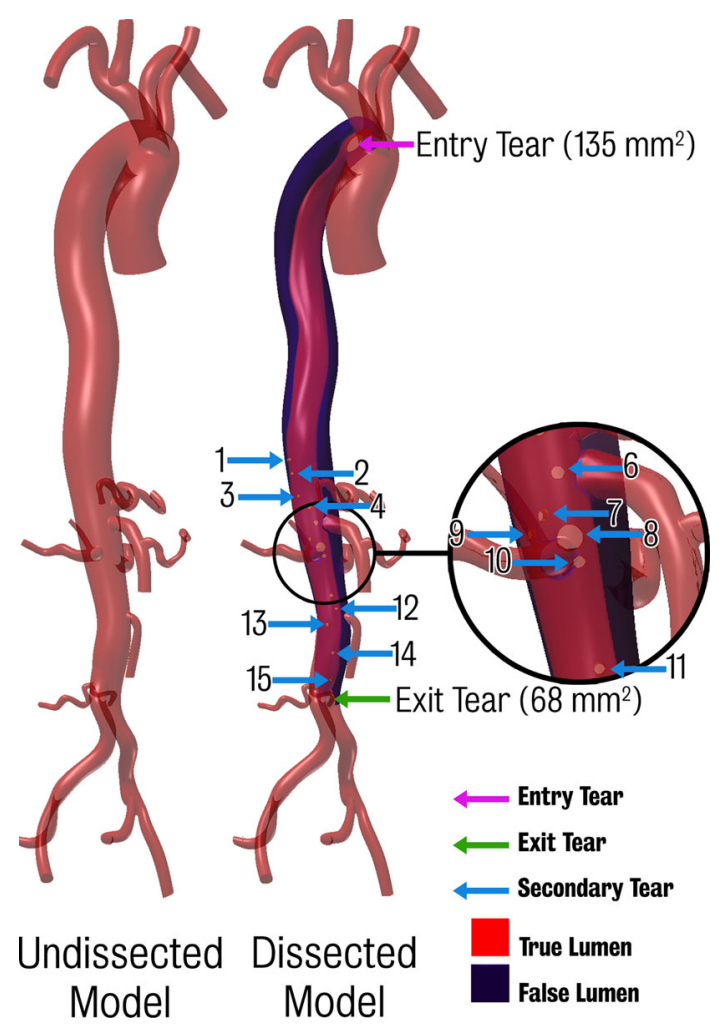

Fig. 3 Undissected and dissected models. Highlighted are the entry tear, exit tear and 15 secondary tears

keeping the outer boundary of its contours, thereby assuming that there are no significant changes in diameter between dissected and undissected models. Both dissected and undissected models have dimensions within the normal anatomical range. The "undissected model" is therefore representative of a healthy aorta and will be used to investigate the acute haemodynamic changes introduced by the dissection. Both these models are shown in Fig. 3. Furthermore, to address the difficulty in detecting connecting tears between the lumina, and to investigate their potential impact on the haemodynamics, two additional dissected models featuring different numbers of secondary connecting tears were also created. These models will be discussed in more detail in Sect. 3 .

The models created in this study are arguably the most complex and geometrically sophisticated aortic models to date. Previous studies of dissection did not include the branching vessels around the coeliac trunk region (Karmonik et al. 2008, 2011a; Alimohammadi et al. 2014). It has however been noted that in the vicinity of branches there exists highly complex flow fields which may have a significant influence on perfusion (Shahcheraghi et al. 2002; Tse et al. 2011). Therefore, the models presented here were constructed to preserve as much of the branching vasculature thought necessary for accurate modelling, resulting in 17 outlets in total, including upper branch vessels (left 

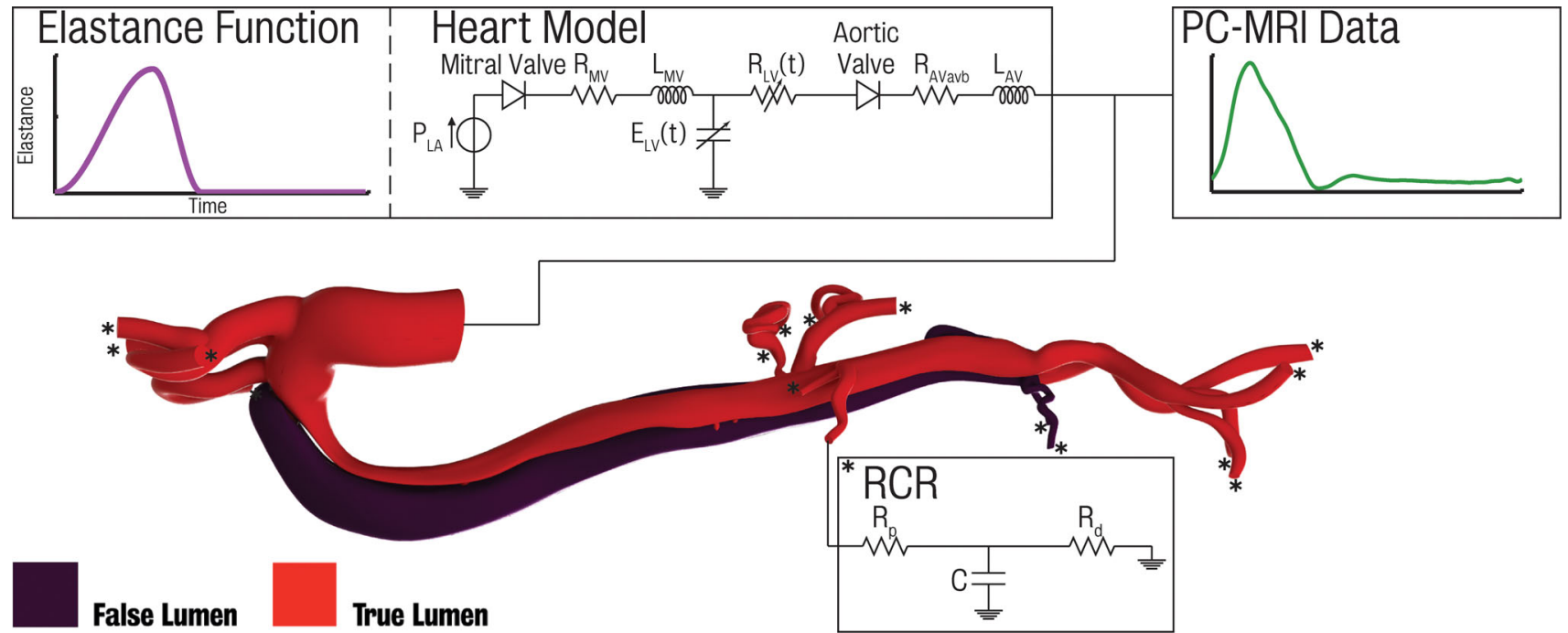

Fig. 4 Schematic showing inlet and outlet boundary conditions used in this paper. A three-element Windkessel lumped-parameter model was specified on each outlet face of the arterial model. Inflow conditions

and right subclavian and carotid arteries), visceral vessels (coeliac trunk, superior mesenteric, inferior mesenteric, left and right renal arteries) and iliac vessels (left and right internal and external iliac).

\subsection{Computational fluid dynamics}

\subsubsection{Coupled multi-domain method for blood flow simulation}

In this section, we describe the coupled multi-domain method for blood flow simulation used in this paper. This method uses a stabilised finite element formulation to solve the incompressible Navier-Stokes equations in a 3D rigid domain reconstructed from image data, coupled to simpler, reducedorder models of the circulation at the inlet and outlet faces of the computational domain. It therefore represents a multiscale (or multi-resolution) approach to blood flow modelling, as flow and pressure are calculated over the entire systemic circulation. Details of this method can be found elsewhere (Vignon-Clementel et al. 2006). For the sake of conciseness, we present the strong form as

$$
\rho \dot{\boldsymbol{v}}+\rho \boldsymbol{v} \cdot \nabla \boldsymbol{v}=-\nabla p+\nabla \cdot \boldsymbol{\tau}
$$$$
\nabla \cdot \boldsymbol{v}=0
$$

where $\rho$ is the blood density (set at $1.060 \mathrm{~kg} / \mathrm{m}^{3}$ for each simulation), $v$ and $p$ and represent the blood velocity and pressure at time $t$ and $\boldsymbol{\tau}=\mu\left(\nabla \boldsymbol{v}+(\nabla \boldsymbol{v})^{\mathrm{T}}\right)$ is the viscous stress tensor of a Newtonian fluid, where $\mu$ is the blood viscosity (set to $4 \times 10^{-3} \mathrm{~Pa} \mathrm{~s}$ ). Body forces are set to zero. With appropriate choices for boundary conditions, and for the solu- were prescribed from either 2D PC-MRI data or via a lumped-parameter heart model. Tuning the elastance function the heart model allows for a close match with the measured 2D PC-MRI flow data

tion and test functional spaces $S, W$ and $P$, the strong form yields the following weak form:

$$
\begin{aligned}
& \int_{\Omega}\{\boldsymbol{w} \cdot(\rho \dot{\boldsymbol{v}}+\rho \boldsymbol{v} \cdot \nabla \boldsymbol{v})+\nabla \boldsymbol{w}:(-p \boldsymbol{I}+\boldsymbol{\tau})-\nabla q \cdot \boldsymbol{v}\} \mathrm{d} V \\
& \quad+\int_{\Gamma_{g}} q \boldsymbol{v} \cdot \boldsymbol{n} \mathrm{d} A-\int_{\Gamma_{h}} \boldsymbol{w} \cdot \boldsymbol{t}^{h} \mathrm{~d} A+\int_{\Gamma_{h}} q \boldsymbol{v} \cdot \boldsymbol{n} \mathrm{d} A+\mathrm{Stab}=0 \\
& \forall \boldsymbol{x} \in \Omega, \forall \boldsymbol{t} \in[0, T]
\end{aligned}
$$

where $\boldsymbol{w} \in W$ and $q \in P$ are test functions for the momentum and mass conservation equations, respectively. $\Omega$ represents the 3D domain generated from the CT data, respectively. $\Gamma_{g}$ is a Dirichlet boundary (typically the inflow) where the test function $\boldsymbol{w}$ vanishes and $\Gamma_{h}$ is a Neumann boundary where a traction

$\boldsymbol{t}^{h}=(-p \boldsymbol{I}+\boldsymbol{\tau}) \cdot \boldsymbol{n}=\boldsymbol{h}(\boldsymbol{v}, p, \boldsymbol{x}, t)$

is prescribed via the 3D-0D domain interface coupling conditions. Figure 4 shows a schematic of the boundary conditions utilised in this paper. The term "Stab" refers to the stabilisation terms of the SUPG formulation (Whiting and Jansen 2001) utilised in our in-house software CRIMSON (www. crimson.software 2015).

A three-element Windkessel model was coupled to the outlet face of each of the branches. In this model, the distal vasculature (small arteries and arterioles) is represented via a zero-dimensional electric circuit analogue that uses resistors to represent haemodynamic resistance and capacitors to capture the vessel compliance (Formaggia et al. 1999; Westerhof et al. 2009; Vignon-Clementel et al. 2010). This simple 
model is one of the most commonly used approaches for outflow boundary condition specification in the simulation of haemodynamics (Stergiopulos et al. 1992; Alastruey et al. 2007; Xiao et al. 2013). Representation of realistic flow and pressure waveforms is achieved via proper specification of the model parameters. The three-element Windkessel model relates flow $Q$ and mean pressure $P$ over a cross section via the following operator:

$$
\left[\begin{array}{c}
P_{d}-P_{c} \\
C \frac{\mathrm{d} P_{\mathrm{d}}}{\mathrm{d} t}
\end{array}\right]=\left[\begin{array}{c}
R_{p} Q \\
Q-\frac{P_{\mathrm{c}}-P_{\mathrm{d}}}{R_{\mathrm{d}}}
\end{array}\right]
$$

where $C$ is the compliance of the distal vasculature, $R_{\mathrm{p}}$ and $R_{\mathrm{d}}$ are the proximal and distal resistances, respectively, $P_{\mathrm{d}}$ is a distal reference pressure (ground value in the Windkessel model), $P_{\mathrm{C}}$ is the Windkessel mid-pressure and $Q$ is the flow going through the vessel. Stabilisation at the 3D-0D interface is provided by a flux term on outflow/inflow boundary of the 3D domain on an element-by-element basis as described in Hughes and Wells (2005).

Regarding the inflow conditions, two different approaches were adopted:

1. An inflow waveform was prescribed at the inlet face of the model. The waveform was derived from the 2D PCMRI data measured just distally to the aortic valve and was mapped to a time-varying parabolic velocity profile.

2. A lumped-parameter model of the left heart tuned to match the flow waveform reconstructed from PC-MRI.

A number of reduced-order heart models have been previously proposed (Hunter et al. 2003; Ottesen et al. 2004; Formaggia et al. 2006; Kerckhoffs et al. 2006; Kim et al. 2009). The one presented in this paper is an implementation of that developed by Lau and Figueroa (2015). This model consists of a constant pressure source $\left(P_{\mathrm{LV}}\right)$ representing the left atrium, a time-varying capacitor representing the left ventricular elastance $\left(E_{\mathrm{LV}}(t)\right)$ and diodes representing the mitral and aortic valves. The pressure in the left ventricle was calculated as a function of the elastance $E_{\mathrm{LV}}$, left ventricular volume $V_{\mathrm{LV}}$ and the unstressed left ventricular volume $V_{\mathrm{u}, \mathrm{LV}}$,

$P_{\mathrm{LV}}(t)=E_{\mathrm{LV}}(t)\left(V_{\mathrm{LV}}(t)-V_{\mathrm{u}, \mathrm{LV}}\right)$

Our model for the ventricular elastance $E_{\mathrm{LV}}(t)$ is based upon trigonometric functions, as detailed in Pope et al. (2009). The model also includes a time-varying resistance $R_{\mathrm{LV}}$ that accounts for viscous pressure losses. This resistance is proportional to a constant $k_{\mathrm{LV}}$, the left ventricular pressure $P_{\mathrm{LV}}$ and the left ventricular flow $Q_{\mathrm{LV}}$ :

$R_{\mathrm{LV}}(t)=k_{\mathrm{LV}} P_{\mathrm{LV}} Q_{\mathrm{LV}}$
The heart model was implemented in the context of the variational multi-scale approach of the multi-domain method (Vignon-Clementel et al. 2006) where 0D models are implicitly incorporated into the weak form of the problem. This leads to a monolithic implementation where the boundary integrals containing the reduced-order models contribute to the left hand-side matrix, and iterations continue until adequate convergence for the coupled problem is achieved (typically 4-6 nonlinear iterations per time step). By tuning the parameters of the heart model, specifically the time to maximum elastance and the maximum elastance value, it is possible to adjust the resulting inflow wave form to produce the desired levels of peak and mean flow. Thus, this approach enables us to reproduce a measured flow waveform without imposing it directly. In doing so, and by using standard values for the heart model published in the literature, we have a method that enables the estimation of cardiac workload, which will make it possible to estimate alterations in cardiac stroke work for this patient when changes in afterload resulting from the sudden onset of the dissection morphology occur (Lau and Figueroa 2015).

\subsubsection{Mesh generation and time integration strategy}

In blood flow modelling, velocity patterns, wall shear stress and pressure gradients can be greatly affected by coarse meshes that ultimately render the blood unphysical numerical viscosity (Taylor and Figueroa 2009). In this work, a two-step strategy was used for the finite element mesh generation. An initial mesh was created for each model using boundary layer and local curvature-based refinement. Simulations were run using the 2D PC-MRI flow data as inflow boundary conditions, and followed by a field-driven mesh adaptation technique to refine the mesh in areas of large velocity gradients (Sahni et al. 2006). The final unstructured field-adapted meshes for each model ranged between 2.6 million tetrahedral elements and 0.4 million nodes in the "undissected model" to 13.2 million tetrahedral elements and 2.4 million nodes in the "dissected model".

The generalised- $\alpha$ method time integrator was used to advance the solution in time (Jansen et al. 2000). Analyses were run using between 2600 and 10,400 time steps per cycle (time step size between $500 \mu \mathrm{s}$ and $125 \mu \mathrm{s}$ for a cardiac cycle of $1.3 \mathrm{~s}$ ) on 256 cores of a 640 core SGI Altix-UV HPC with Nehalem-EX architecture. Residual control, which limits the sum total of all the individual nodal residuals for the entire mesh, was set to $1 \times 10^{-3}$. Each cardiac cycle took approximately between 4 and $18 \mathrm{~h}$ to run. For each case, simulations were run between 3 and 7 cardiac cycles until a cycle-to-cycle periodic solution in flow and pressures was achieved. 


\section{Results}

\subsection{Study 1: Haemodynamic alterations in aortic dissection}

\subsubsection{Baseline haemodynamics in undissected aorta}

The simulation considered first was that of the undissected aorta model created from the aortic dissection image data by virtually removing the septum while keeping all aortic contours unchanged. As no patient-specific data were available for the undissected state, the same flow data derived from the dissected 2D PC-MRI study were applied at the inlet. The parameters of each three-element Windkessel model were manually tuned to give typical flow and pressure waveforms at the outlets (Xiao et al. 2014). The values of these parameters are given in Table 4.

Figure 5 shows pressure and flow wave forms at selected outlets as well as visualisations of pressure and flow in the aorta at peak systole. The results show physiologically realistic ranges for flow, pressure and flow splits between different vascular regions.

\subsubsection{Dissected aorta}

Following the simulation of the undissected model, flow through the dissected model was investigated using the same inlet and outlet boundary conditions as in the preceding analysis. From the three 2D PC-MRI acquisitions in the descending aorta, it was known that mean total flow (for both true and false lumina) ranges between of 55.6 and $50.3 \mathrm{~cm}^{3} / \mathrm{s}$. It was found that the simulated flow down the descending dissected aorta lied within these limits without having to change any of the inflow and outflow boundary conditions relative to the undissected model.

Figure 6 shows haemodynamics in the dissected aorta at peak systole. Significant changes are apparent relative to the results depicted in Fig. 5. In terms of pressure at the different branches, there is a clear increase in pulse pressure at the aortic inflow and the upper branches. The systolic and diastolic left carotid pressure are $146 / 75 \mathrm{mmHg}$ for the undissected case and 159/71 mmHg for the dissected case. The mean pressure for the ascending vessels remains relatively constant. Flow is increased to these branches. Specifically, flow to the left common carotid increased by $14.8 \%$ in the dissected case. The vessels in the distal descending aorta experienced drops in both mean and pulse pressure. For instance, the right external iliac had a mean and pressure of $94 \mathrm{mmHg}$ and systolic and diastolic pressures of 151/76 $\mathrm{mmHg}$ in the undissected case, whereas the mean, systolic and diastolic pressures for the dissected case are 90 and $147 / 72 \mathrm{mmHg}$. Several other outlets experienced changes in pressure and flow, for example, in the SMA mean pressure dropped by $6.5 \%$ and mean flow by $5.3 \%$. Flow to the vessels branch-
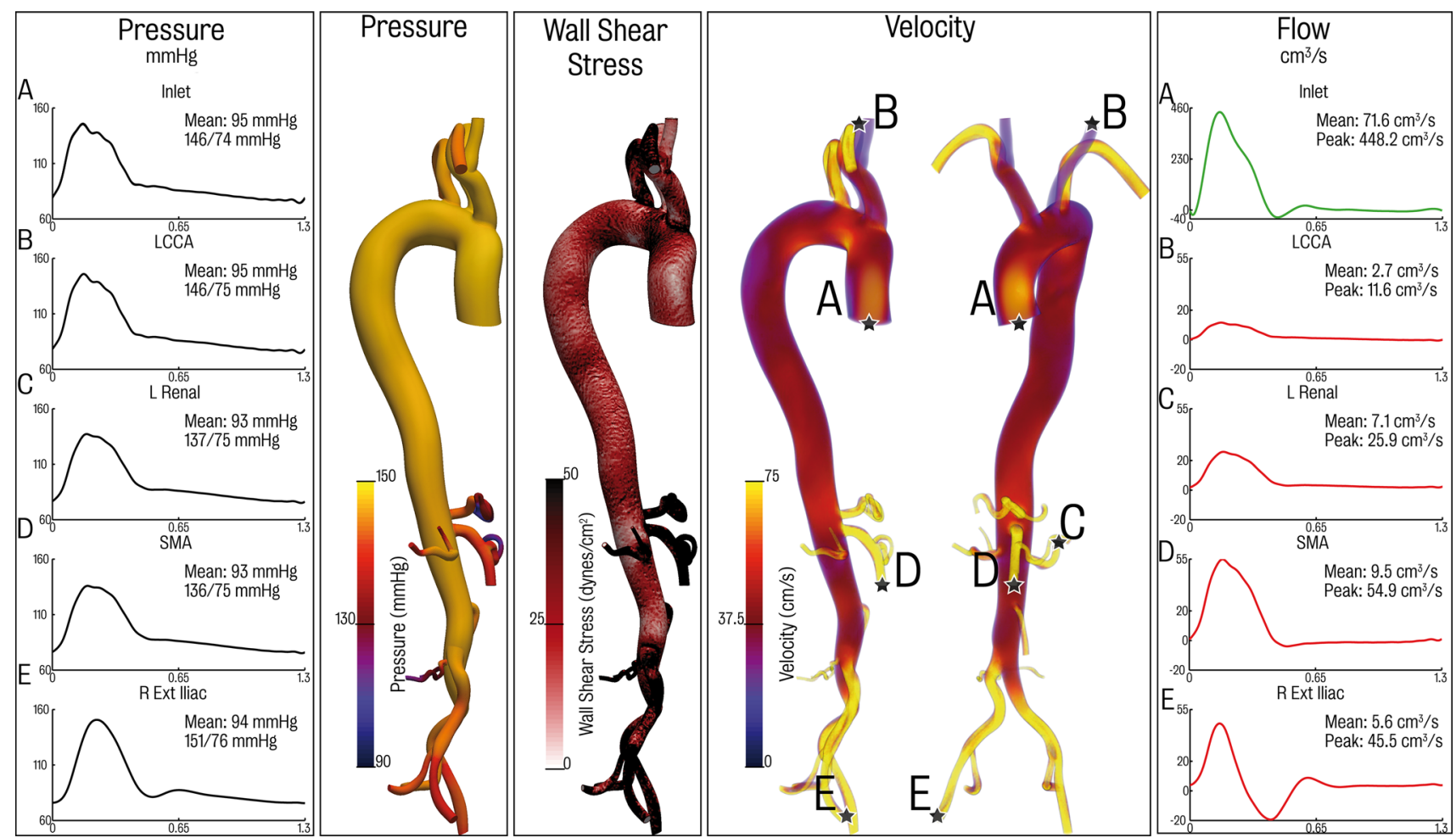

Fig. 5 Visualisations of pressure, wall shear stress and velocity at peak systole for the baseline undissected model. Shown also are pressure and flow waveform plots at the inlet, left common carotid, left renal, superior mesenteric artery and right external iliac 
Table 4 RCR parameters tuned to produce physiologic flow and pressure waveforms for the undissected aortic model

\begin{tabular}{llll}
\hline & $\mathrm{R}_{\mathrm{p}}\left[\mathrm{g} /\left(\mathrm{mm}^{4} \mathrm{~s}\right)\right]$ & $\mathrm{C}\left(\mathrm{mm}^{4} \mathrm{~s}^{2} / \mathrm{g}\right)$ & $\mathrm{R}_{\mathrm{d}}\left[\mathrm{g} /\left(\mathrm{mm}^{4} \mathrm{~s}\right)\right]$ \\
\hline RCCA & 0.80 & 6.10 & 4.53 \\
RSCA & 0.13 & 7.64 & 1.89 \\
LCCA & 0.78 & 6.14 & 4.40 \\
LSCA & 0.12 & 7.87 & 1.72 \\
Hepatic & 0.24 & 1.15 & 4.06 \\
Splenic & 0.14 & 2.04 & 2.30 \\
M Coeliac & 0.23 & 1.18 & 3.95 \\
SMA & 0.13 & 3.87 & 1.15 \\
R Renal 1 & 0.48 & 2.37 & 1.94 \\
R Renal 2 & 1.11 & 1.19 & 4.45 \\
L Renal & 0.32 & 3.34 & 1.30 \\
IMA & 0.68 & 0.73 & 6.13 \\
M Sacral 1 & 0.54 & 0.52 & 9.04 \\
M Sacral 2 & 0.78 & 0.36 & 13.12 \\
L Int Iliac & 0.10 & 0.15 & 3.87 \\
R Int Iliac & 0.13 & 0.15 & 5.15 \\
L Ext Iliac & 0.06 & 0.49 & 2.49 \\
R Ext Iliac & 0.06 & 0.55 & 2.18 \\
\hline
\end{tabular}

ing from the coeliac trunk dropped by a total of $13.5 \%$ and pressure by $13.6 \%$.

Locally, there are significant alterations in haemodynamics, particularly around the entry tear, true lumen and coeliac trunk regions. Some of these changes are highlighted in Fig. 7. The spatial distribution of pressure in the dissected model at peak systole around the entry tear ranges between 58.6 and $161.0 \mathrm{mmHg}$. This range was much smaller in the corresponding region of the undissected model: 92.2$148.0 \mathrm{mmHg}$. The peak systolic pressure gradient along the length of the true lumen is larger than that seen in the corresponding location of the undissected aorta: from $160 \mathrm{mmHg}$ at the entry tear to $135 \mathrm{mmHg}$ at the exit tear in the dissected model. Correspondingly, the undissected model has a pressure gradient of $5 \mathrm{mmHg}$ in the descending aorta with pressures in the range of $145-140 \mathrm{mmHg}$. Pressure in the proximal portion of the dissection is generally higher in the true lumen than in the false. The reverse trend is observed in the distal portion. This pattern has also been noted in other studies (Tse et al. 2011; Alimohammadi et al. 2014). An area of marked increased pressure (over $160 \mathrm{mmHg}$ ) is observed where flow through the entry tear impinges on the wall of the false lumen. This localised region of high blood pressure
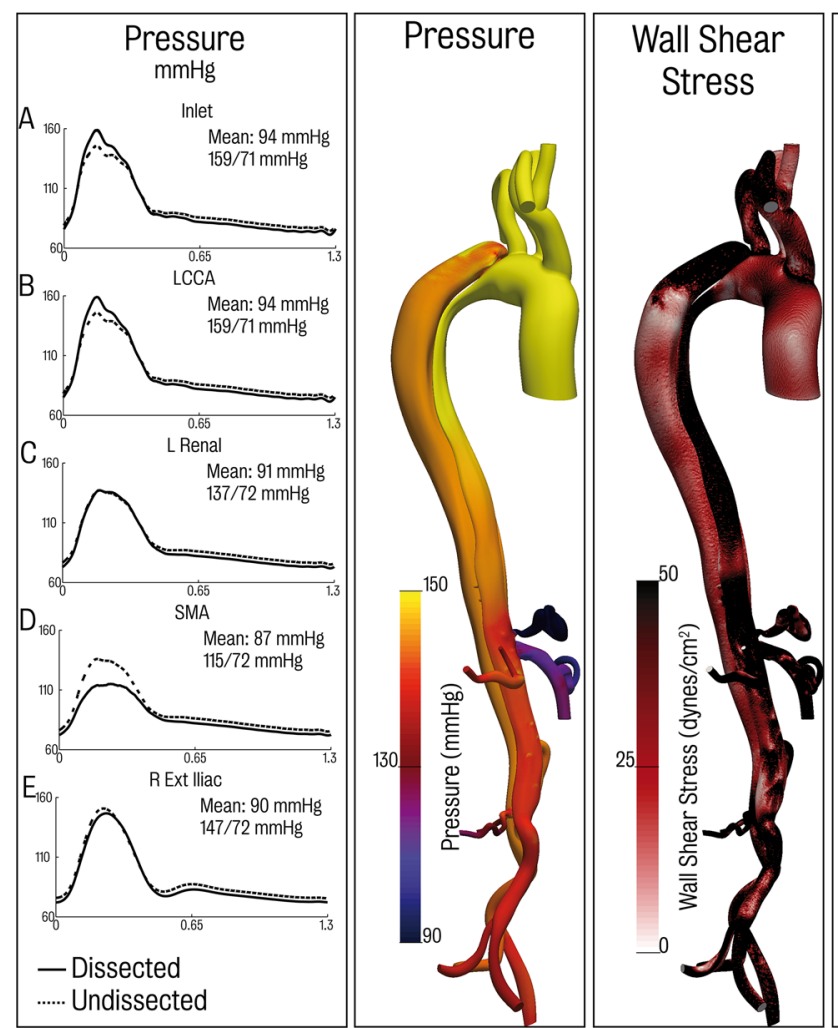

Fig. 6 Visualisations of pressure, wall shear stress and velocity at peak systole for the baseline dissected model at peak systole. Shown also are comparative pressure and flow waveform plots at the inlet, left common

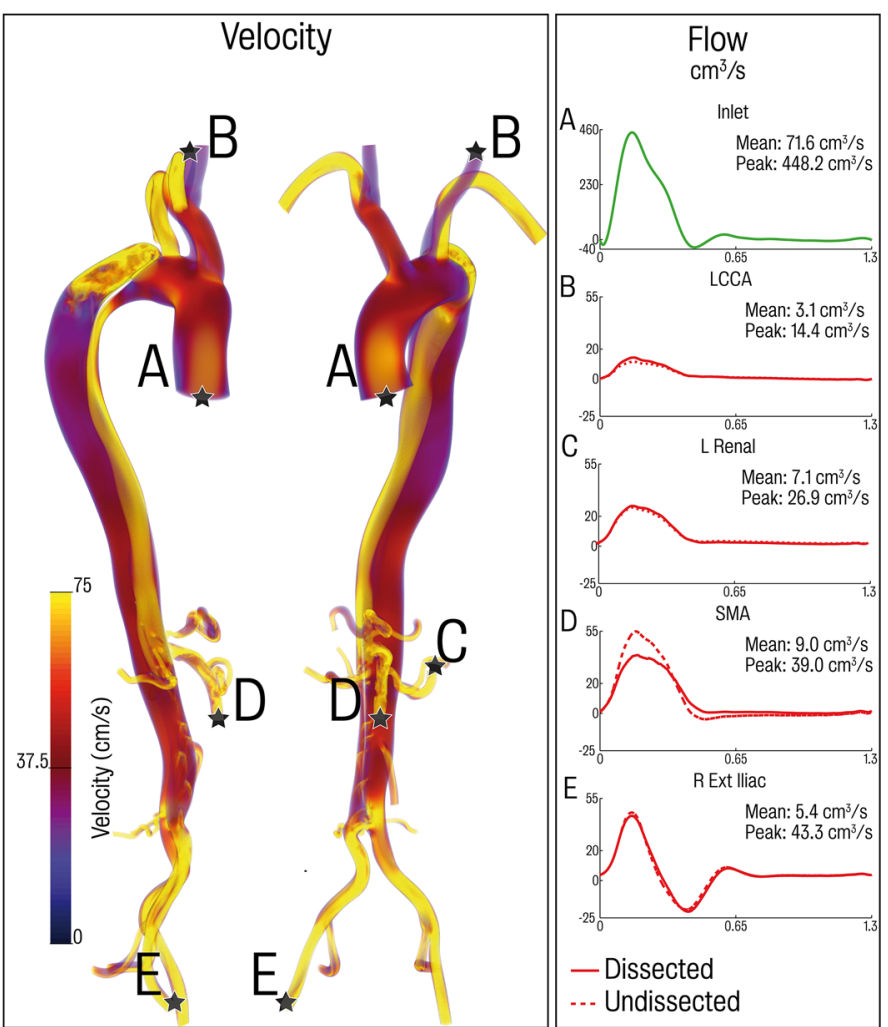

carotid, left renal, superior mesenteric artery and right external iliac for both the undissected and dissected models 

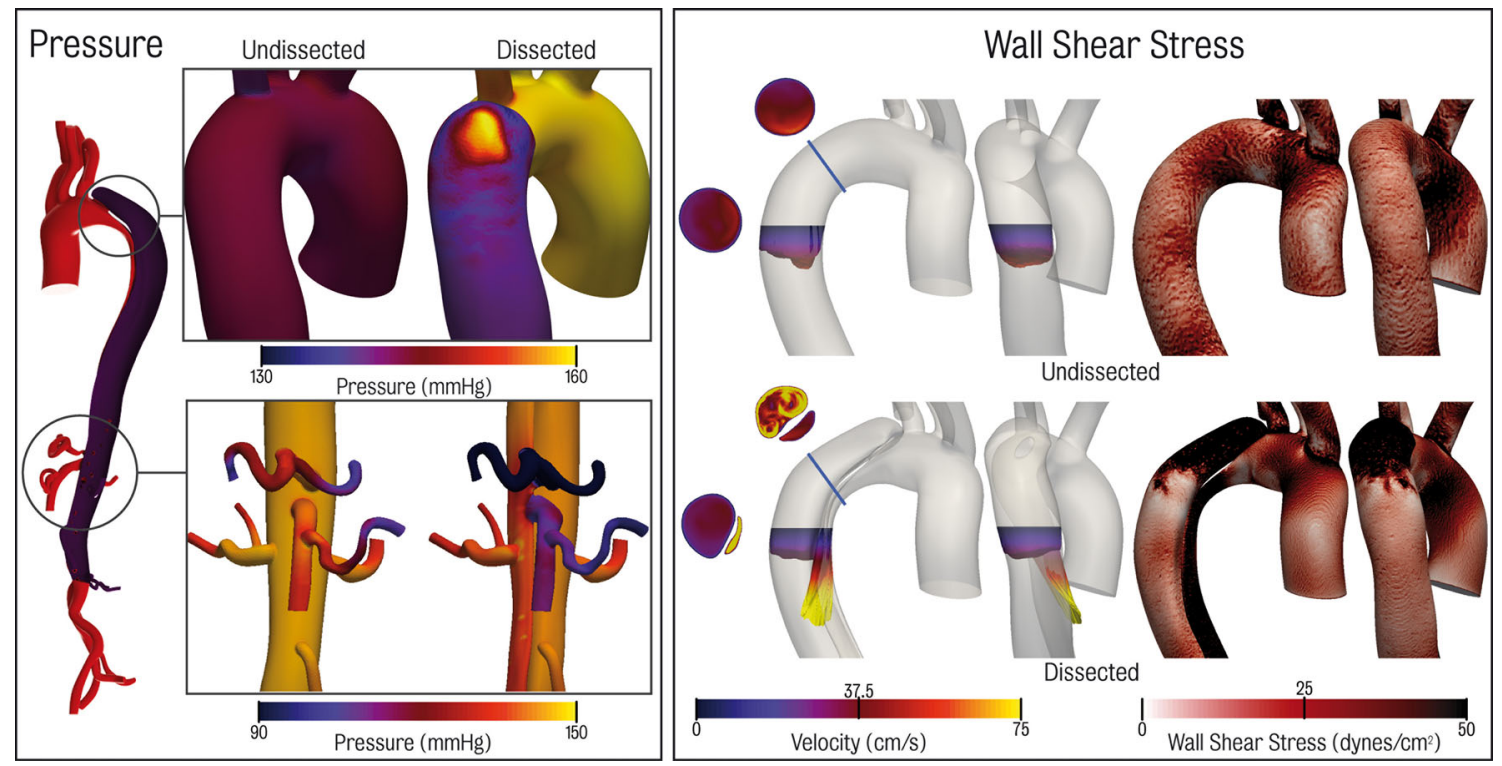

Fig. 7 Localised regions of haemodynamic change in the presence of dissection comparing pressure and wall shear stress at peak systole between the dissected and undissected models

on the structurally compromised false lumen could lead to enlargement and potential rupture (Tsai et al. 2007; Chen et al. 2013).

The dissection case shows large true lumen peak velocities in the range of $70 \mathrm{~cm} / \mathrm{s}$ in the coeliac trunk region where there is a large flow demand. Distal to the coeliac trunk region, there is a significant drop in peak velocities. The computed values of $35 \mathrm{~cm} / \mathrm{s}$ are comparable with those seen in the undissected case.

Highly complex flow patterns are observed in the false lumen in the entry tear region. A dramatic increase in timeaveraged wall shear stress (TAWSS) from approximately 5 dynes $/ \mathrm{cm}^{2}$ in the undissected case to over 100 dynes $/ \mathrm{cm}^{2}$ in the dissected case is observed. A more moderate increase in true lumen TAWSS is observed in the coeliac trunk region, going from 5 to 10 dynes $/ \mathrm{cm}^{2}$ in the undissected case to over 20 dynes $/ \mathrm{cm}^{2}$ in the dissected case.

\subsubsection{Comparison between computed and measured flows in the descending aorta}

In this section, we compare the computed flows in the true and false lumina with those measured using PC-MRI at three different locations in the descending thoracic aorta. For clarity, these shall be referred to as proximal, medial and distal locations. Figure 8 shows quantitative comparisons of PCMRI and simulation data at each location.

There is good agreement in mean flows in true and false lumina between simulation and imaging data. The MRI gives a total flow (e.g. sum of false lumen and true lumen flow) at each location of $55.6,53.8$ and $50.3 \mathrm{~cm}^{3} / \mathrm{s}$ respectively. The computation predicts a uniform value for the total flow of $51.4 \mathrm{~cm}^{3} / \mathrm{s}$ at each location, resulting in relative errors between measurement and simulation of $-7.6,-4.5$ and $+2.2 \%$, respectively. It is important to note that this good agreement in total mean flow between data and simulations was not imposed in any way, but rather the result of properly tuned outflow boundary conditions in the remaining branches of the model as these dictated the flow splits to the different branches, as well as a realistic reconstruction of the aortic anatomy. On average, the false lumen carried $79 \%$ of the total descending aortic flow according to the MRI, whereas in the simulation this value was $76 \%$.

Examining each lumen individually, MRI false lumen mean flows at each location are $40.6,42.5$ and $42.4 \mathrm{~cm}^{3} / \mathrm{s}$, whereas the simulations produce a nearly uniform mean value of $38.7 \mathrm{~cm}^{3} / \mathrm{s}$ at all three locations, resulting in relative errors of $-4.7,-8.9$ and $-8.7 \%$, respectively. Considering the true lumen, MRI mean flows are $15.1,11.1$ and $7.9 \mathrm{~cm}^{3} / \mathrm{s}$ at the location, while the computations show a constant value of $12.7 \mathrm{~cm}^{3} / \mathrm{s}$ for all three locations and relative errors of $-15.9,+14.4$ and $+60.8 \%$, respectively. The larger relative errors in true lumen flow can be explained by the smaller mean values recorded at this location. On average, MRI data show the true lumen carries $21 \%$ of total flow, whereas in the simulation this value is $25 \%$. It is important to note that PC-MRI data were acquired with single VENC, but as peak velocities were significantly lower in the false lumen than in the true lumen (in the order of $50 \%$ less), true lumen and false lumen flows may have differed somewhat from what was recorded.

Overall, the small differences in flow between the three locations are to be expected due to the close proximity of the different slices $(11 \mathrm{~cm}$ between proximal and distal 


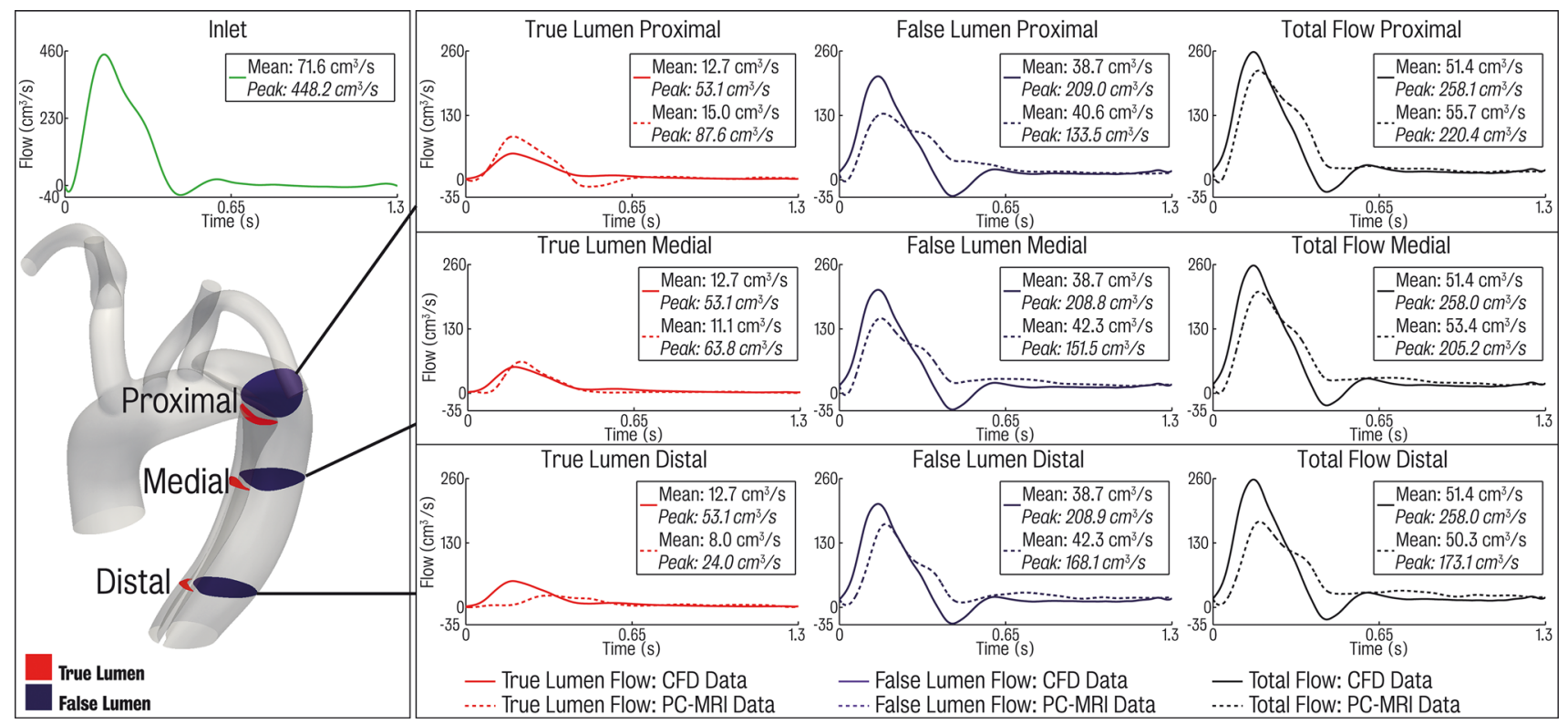

Fig. 8 Comparison between 2D PC-MRI and CFD simulation data for the true lumen, false lumen and total flow. True lumen is shown in red, false lumen in purple and the total flow in black. CFD data are shown by the solid lines, while 2D PC-MRI data are presented in the dashed lines

slices) and the apparent lack of secondary connecting tears or branching vessels between the proximal and distal slices. The increase in measured false lumen flow from the proximal to the distal location ( 40.6 vs. $42.3 \mathrm{~cm}^{3} / \mathrm{s}$ ) could suggest the presence of a connecting tear. However, this could not be located in neither the CT data nor the 4D-PC-MRI data. In the simulation data, this lack of connecting tears results in identical flows at each location due to the rigid wall and blood incompressibility assumptions. In Sect. 3.3, we study the impact that additional connecting tears between lumina may have in the aortic haemodynamics.

Despite the mean flows being relatively well matched between PC-MRI and simulated data, there exists a greater discrepancy when considering the systolic and diastolic range at each location. In the true lumen at two of the three locations, the peak-to-peak range of flows from the simulated data underestimated that of the PC-MRI data, while in all three false lumen locations the amplitude of the simulated waveforms is larger than that of the PC-MRI data. PCMRI data for the true lumen have a systolic/diastolic range of $-13.8 / 87.6,0.8 / 63.4$ and $0.6 / 24.0 \mathrm{~cm}^{3} / \mathrm{s}$ at the respective locations, while the simulated data at the same locations have a range of $1.9 / 53.1,1.9 / 53.1$ and $1.9 / 53.1 \mathrm{~cm}^{3} / \mathrm{s}$. In the false lumen, the PC-MRI data have a range of $133.5 / 10.3,151.5 / 14.9$ and 168.1/10.1 at the respective locations, while the corresponding simulated data show ranges of 209.0/-32.7, 208.8/-32.8 and 208.9/-32.8. These discrepancies may be explained by the lack of compliance in the rigid 3D aortic model. In reality, the vessel walls are compliant and the septum deforms significantly, especially in an acute setting. The arterial compliance enables the vessel to accom- modate part of the blood volume during systole, reducing the peak flow rates through a given section. In early diastole, the stored blood volume is released, increasing minimum flow during diastole. A rigid 3D model cannot capture this effect however, and the only compliance in the computational model is provided by the capacitor component in the $0 \mathrm{D}$ Windkessel model coupled to each outlet. This is supported by the observed of higher peak-to-peak flows of the simulated to PC-MRI data and is expected when using a rigid model.

\subsection{Study 2: Analysis of the impact of the flap on left ventricular function}

In this study, a lumped-parameter heart model was coupled to the inlet face of the dissected and undissected models to examine changes in stroke work induced by the dissection. The heart model enables the quantification of left ventricular volume and pressure throughout the cardiac cycle. From these data, a pressure volume loop can be generated to quantify the cardiac workload. The cardiac output, measured with MRI in the dissected aorta, was assumed to be identical for both dissected and undissected cases. This is a reasonable first approximation, as the heart is known to adjust to changes in afterload to maintain a relatively constant output that satisfies the metabolic demands (Lau and Figueroa 2015). The outflow boundary conditions were kept unchanged. The elastance function characterising the performance of the heart model was adjusted in both cases to match the measured flow waveform at the level of the aortic root. The iterative process sought to match mean cardiac output, systolic cardiac output, cardiac cycle and length of systolic phase. The 
Table 5 Tuned parameters of the heart model for the dissected and undissected models

\begin{tabular}{lll}
\hline & Dissected model & Undissected model \\
\hline Period $(\mathrm{s})$ & 1.3 & 1.3 \\
End diastolic volume $\left(\mathrm{mm}^{3}\right)$ & 130,000 & 130,000 \\
Unstressed volume $\left(\mathrm{mm}^{3}\right)$ & 0.0 & 0.0 \\
Preload $\left(\mathrm{g} / \mathrm{mm}^{2}\right)$ & 533.320 & 533.320 \\
Time to maximum elastance $(\mathrm{s})$ & 0.410 & 0.420 \\
Time to relaxation $(\mathrm{s})$ & 0.205 & 0.210 \\
Maximum elastance $\left(\mathrm{g} /\left(\mathrm{mm}^{4} \mathrm{~s}^{2}\right)\right)$ & 0.460 & 0.400 \\
Minimum elastance $\left(\mathrm{g} /\left(\mathrm{mm}^{4} \mathrm{~s}^{2}\right)\right)$ & $4.102 \times 10^{-3}$ & $4.102 \times 10^{-3}$ \\
Aortic valve resistance $\left[\mathrm{g} /\left(\mathrm{mm}^{4} \mathrm{~s}\right)\right]$ & $1.0 \times 10^{-5}$ & $1.0 \times 10^{-5}$ \\
Aortic valve inductance $\left[\mathrm{g} / \mathrm{mm}^{4}\right]$ & $1.0 \times 10^{-5}$ & $1.0 \times 10^{-5}$ \\
Ventricular resistance $\left(\mathrm{s} / \mathrm{mm}^{3}\right)$ & $5.0 \times 10^{-7}$ & $5.0 \times 10^{-7}$ \\
Mitral valve Resistance $\left[\mathrm{g} /\left(\mathrm{mm}^{4} \mathrm{~s}\right)\right]$ & $3.9453 \times 10^{-7}$ & $3.9453 \times 10^{-7}$ \\
Mitral valve Inductance $\left(\mathrm{g} / \mathrm{mm}^{4}\right)$ & $1.334 \times 10^{-5}$ & $1.334 \times 10^{-5}$ \\
\hline
\end{tabular}

adjusted parameters were maximum elastance and time to maximum elastance (Table 5). Iterations on maximum elastance and time to maximum elastance parameters stopped when the obtained maximum and mean cardiac output were within $5 \%$ of the measured PC-MRI values.

The left panel of Fig. 9 shows a good match in the waveforms generated via the $0 \mathrm{D}$ heart model and the physiological PC-MRI data. The heart model waveforms for both dissected and undissected cases lack the slight early diastolic back flow observed in the PC-MRI data. This is a direct consequence of the way the aortic valve is modelled in the OD heart model (see Fig. 4): the diode representing the function of the aortic valve closes instantly as soon an adverse pressure gradient between the left ventricle and the aorta is detected. This effectively precludes back flow when using the heart model.

The right panel of Fig. 9 shows the PV loops for both dissected and dissected cases. The four distinct phases of the cardiac cycle (ventricular filling, isovolumetric contraction, ejection and isovolumetric relaxation) as well as the aortic valve opening and closing can be observed in the diagrams. The estimated left ventricular work load, quantified by the area enclosed by the plot, shows a significant increase $(13.7 \%)$ in the dissected case work $(1.9 \mathrm{~kJ})$, relative to the work performed by the heart in the undissected anatomy $(1.7 \mathrm{~kJ})$. The increased in work is the result of an increase in maximum elastance during peak systole due to the higher afterload in the dissected case. The presence of the septum therefore makes the heart work harder to produce the same cardiac output as in the undissected case.

\subsection{Study 3: Sensitivity study of the impact of secondary entry tears}

While the location of some of the tears appeared to be clear in the CTA (see Fig. 10, left), the tear location could not be confirmed by the 4D PC-MRI data, as there was no distinct change in the velocity field that would indicate a connection between the true and false lumina (see Fig. 10, centre and right panels). These panels also show a comparison between CFD and 4D PC-MRI streamlines at peak systole and middiastole. Good qualitative agreement between patterns in the velocity field in data and simulation can be observed: for example, high velocities around the main entry tear and in the true lumen are apparent in both imaging and simulation data. An important question therefore remains, do secondary tears have a significant impact on dissection haemodynamics and is there a better agreement between clinical data (e.g. MR-derived flow) and CFD results when secondary tears are included?

A study was thus designed to investigate the influence of secondary tears on aortic dissection haemodynamics. Two modifications of the 17-tear baseline dissected model were constructed. The first is a "maximal tears" model in which two additional tears in the descending aorta were included. These tears, while evident on the CT data, were not seen in the 4D PC-MRI data. The second is a "minimal tears" model containing only the primary entry and exit tears (see Fig. 11). The final adapted finite element mesh sizes for all three models are given in Table 6.

Comparisons between the 2D PC-MRI data acquired and CFD results at each of the three locations (proximal, medial and distal) for the three dissection models are shown in the top panel of Fig. 12. The computed total flow (defined as the sum of true and false lumen flow) shows a good comparison with the 2D PC-MRI data for all three models, with mean simulation errors ranging from $-3 \%$ (maximal tears) to $-1.4 \%$ (minimal tears). However, when comparing flow through each individual lumen, the minimal tears case shows larger errors than the baseline and maximal tears models: $122.6 \%$ mean error in the true lumen and $-34.7 \%$ error in 


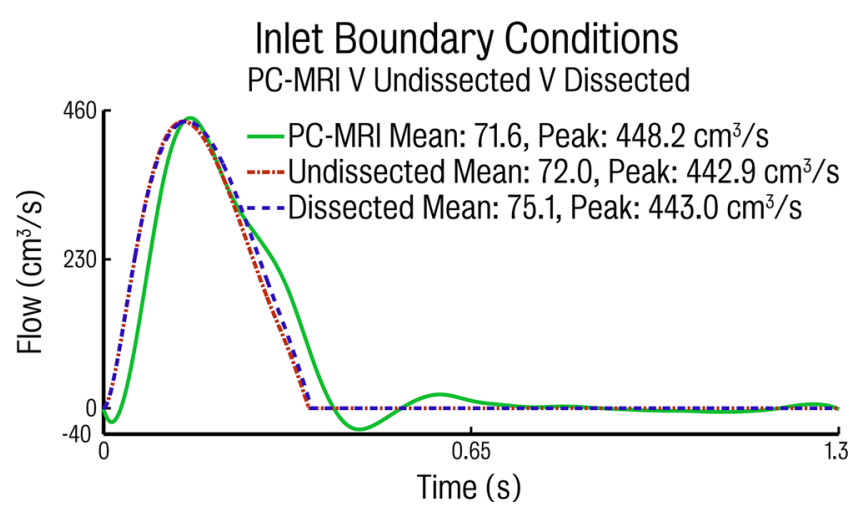

Fig. 9 Left inflow waveforms obtained with PC-MRI and heart model. Right pressure-volume $(P V)$ loops generated via using the heart model for both the undissected and dissected simulations. The PV loops illustrate the mitral valve opening $(A)$, the diastolic ventricular filling $(A-B)$, mitral valve closing $(B)$, isovolumetric contraction $(B-C)$, aortic valve

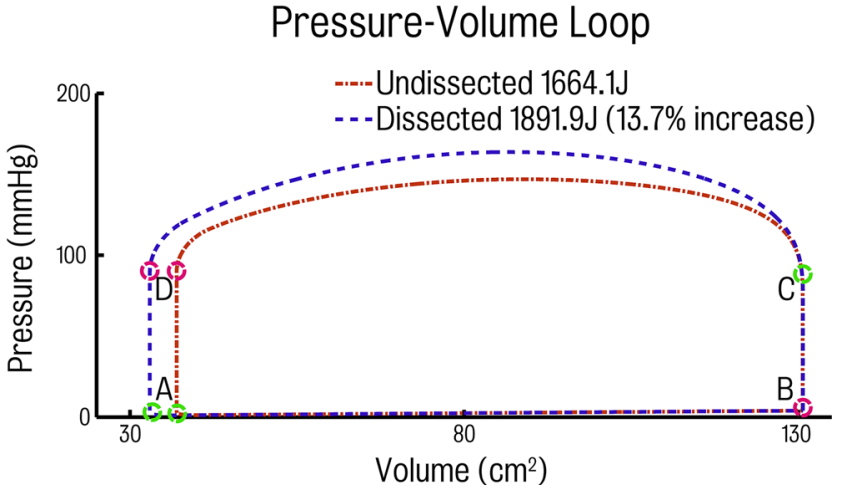

opening $(C)$, ejection $(C-D)$, aortic valve closing $(D)$, followed by isovolumetric relaxation $(D-A)$. The area enclosed by the $P V$ loop represents the left ventricular stroke work. There is an increase in the stroke work of $13.7 \%$ in the presence of dissection

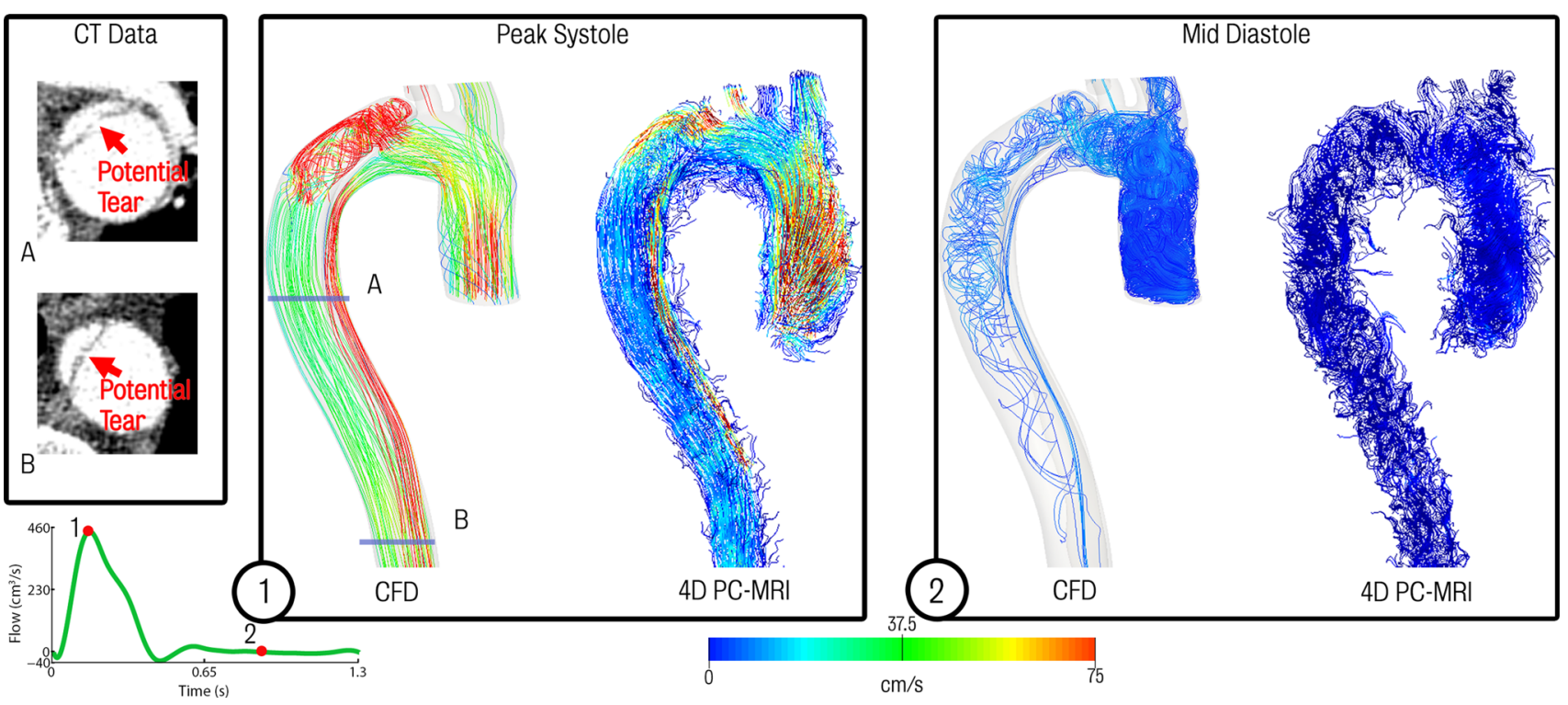

Fig. 10 Comparison of CFD data and acquired 4D PC-MRI flow data at peak systole and mid-diastole. Also shown are the CT image data at two locations showing suspected secondary tears which were not apparent on the 4D PC-MRI data

the false lumen. The baseline and maximal tears models show similar results, and match the 2D PC-MRI data more closely.

The bottom panel of Fig. 12 shows a comparative of flow and pressure waveforms in the region proximal to the coeliac trunk for each of the models. Note that no patient-specific data were acquired in this region, and this comparison is presented to highlight the differences between each simulation. The results show that the baseline dissected and maximal tears cases are relatively comparable, whereas the pressure and flow waveforms in the minimal tears case are significantly different. These differences are more pronounced in true lumen, where large changes in peak $(+81.4 \%)$ and mean $(+87.3 \%)$ flow and peak pressure $(-17.8 \%)$ are observed relative the baseline dissected case. Tears in the septum allow pressure between the true and false lumina to equalise. The differences observed in the true lumen of the minimal tears case are likely a combined effect of the flow demand of the branching vessels in this region having to be supplied solely by the lumen they branch from resulting in increased flow in the true lumen, and Poiseuille's law, as the effective cross-sectional area communicating blood to the true lumen decreases due to the absence of the communicating tears and results in a pressure drop.

These results suggest that in this case some tears do indeed exit in the trunk region given that the minimal tears case shows results clearly different from the baseline and maximal tears, and with higher errors compared to the 2D PC-MRI data. The issue however still remains, as these small sec- 


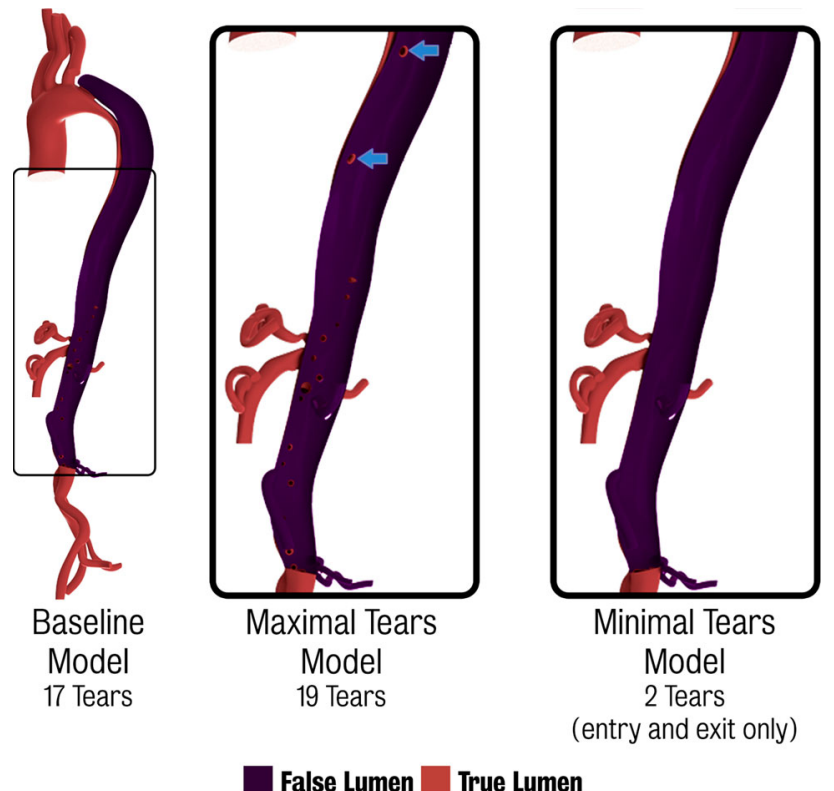

Fig. 11 Left baseline dissected model featuring a total of 17 tears. Centre "maximal tears" model with two additional tears in the descending aorta evident in the CT data but not in 4D PC-MRI. Right "minimal tears" model where all but the primary entry and exit tears were removed

Table 6 Final mesh sizes for each dissected model

\begin{tabular}{ll}
\hline Model & Mesh size $\left(\times 10^{6}\right)$ \\
\hline Baseline dissected & 1.8 nodes $/ 11.8$ elements \\
Maximal tears & 2.4 nodes $/ 13.2$ elements \\
Minimal tears & 1.8 nodes $/ 11.5$ elements \\
\hline
\end{tabular}

ondary tears in a thin and highly distensible dissection flap prove to be very difficult to image.

\section{Discussion}

In this study, we used multiple sources of medical image data (CT; 2D PC-MRI velocity data in the true and false lumina and ascending aorta; and 4D PC-MRI) to inform our computational analysis of aortic dissection. The combination of highly resolved CT and 2D PC-MRI data has allowed, for the first time, the creation of a highly detailed, multi-branch geometric model of the dissected morphology featuring 17 branches. The 2D PC-MRI flow data were used to tune both the inflow and outflow reduced-order models coupled to the 3D domain, and to validate the simulation results.

Building a geometric model of the aortic vasculature involves segmenting the patient's image data to obtain the volume of interest where the blood flow simulation is to be performed. There are several powerful 3D automatic and semiautomatic segmentation tools such as Seg3D (CIBC
2015), MITK (Wolf et al. 2005) and ITK-Snap (Yushkevich et al. 2006) that can perform this task with relatively little effort from the user. However, automatic segmentation may be difficult to apply on images with low resolution or poor signal-to-noise ratio, circumstances unfortunately present in aortic dissection imaging. Indeed, the small size and motion of the septum often make it difficult to delineate the boundaries between true and false lumina. It was found that, after trying several different tools, automatic 3D segmentation methods produced numerous artefacts in the form of "bleeds" between the two lumina (see Fig. 13). A significant amount of time was spent trying to manually correct these artefacts, a task that is non-practical as it was significantly more time-consuming than the segmentation process itself. It was therefore concluded that in cases of aortic dissection, a 2D segmentation approach is preferable. This segmentation approach, although requiring a larger degree of user intervention (i.e. it is less automatic), is more robust in situations of poorly defined features in the image data as the lofting method interpolates the surface between each contour which may be defined at locations where said features are clearly visible and also allows for human judgement to be employed in regions where features may be obscure.

Previous CFD studies in aortic dissection have been somewhat limited due to the assumptions adopted to determine inflow and outflow boundary conditions. For instance, most studies have used flow data acquired from healthy subjects (Tse et al. 2011; Chen et al. 2013; Alimohammadi et al. 2014). Dissection patients are often prescribed the antihypertensive therapies such as beta blockers and morphine sulphate and this may have associated issues. The therapies seek to maintain normal blood pressure, thereby reducing aortic wall stress in dissection patients (Elefteriades et al. 1999; Erbel et al. 2001; Nienaber and Eagle 2003; McGee et al. 2005; Coady et al. 2010). However, cardiac output is often also reduced via reduction in left ventricular contractility. Therefore, assuming healthy inflow conditions may overestimate actual inflow conditions (Chen et al. 2013) in dissection patients. Our paper has used patient-specific data at a number of locations to inform and validate the model. Similarly, with regard to outflow boundary conditions, most studies have either imposed pressure and/or velocities acquired from healthy subjects or have applied zero pressure conditions (Tse et al. 2011; Karmonik et al. 2011a; Chen et al. 2013). In this study, we have used tuned lumped-parameter Windkessel models to represent the resistance and compliance of the distal vasculature.

We performed three studies with two main overriding objectives: i) to validate our computational results against subject-specific flow data, and ii) to obtain a deeper understanding on the alterations in aortic haemodynamics induced by the dissection. 


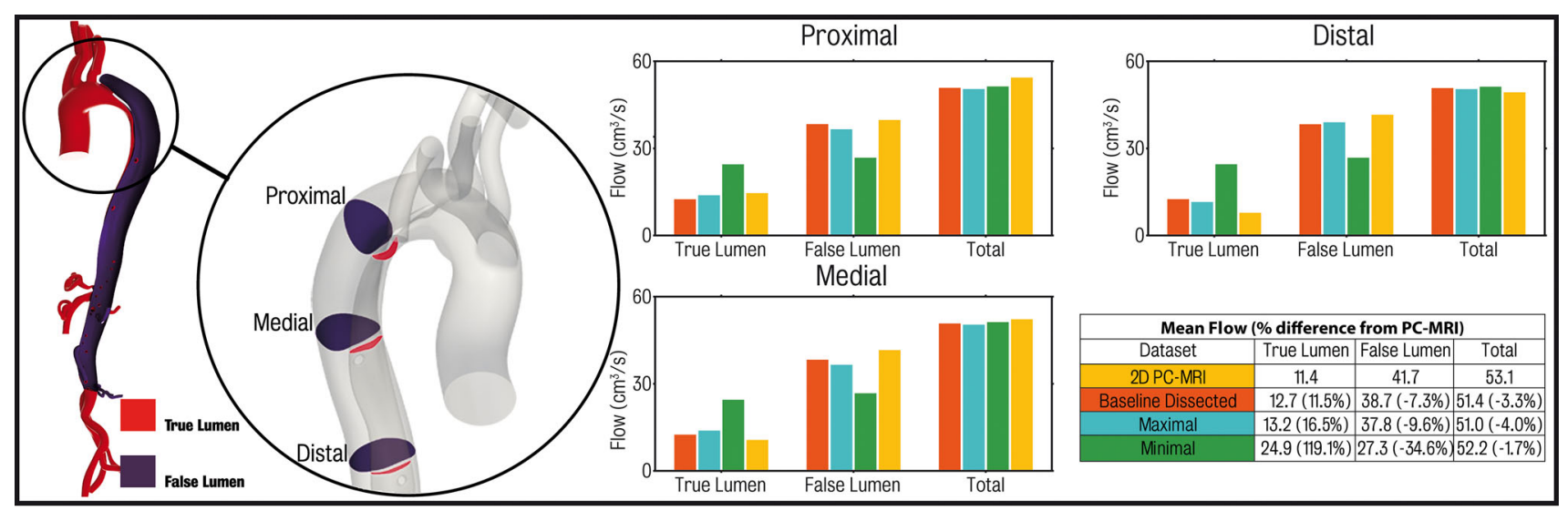

Mean flows at 2D PC-MRI Locations

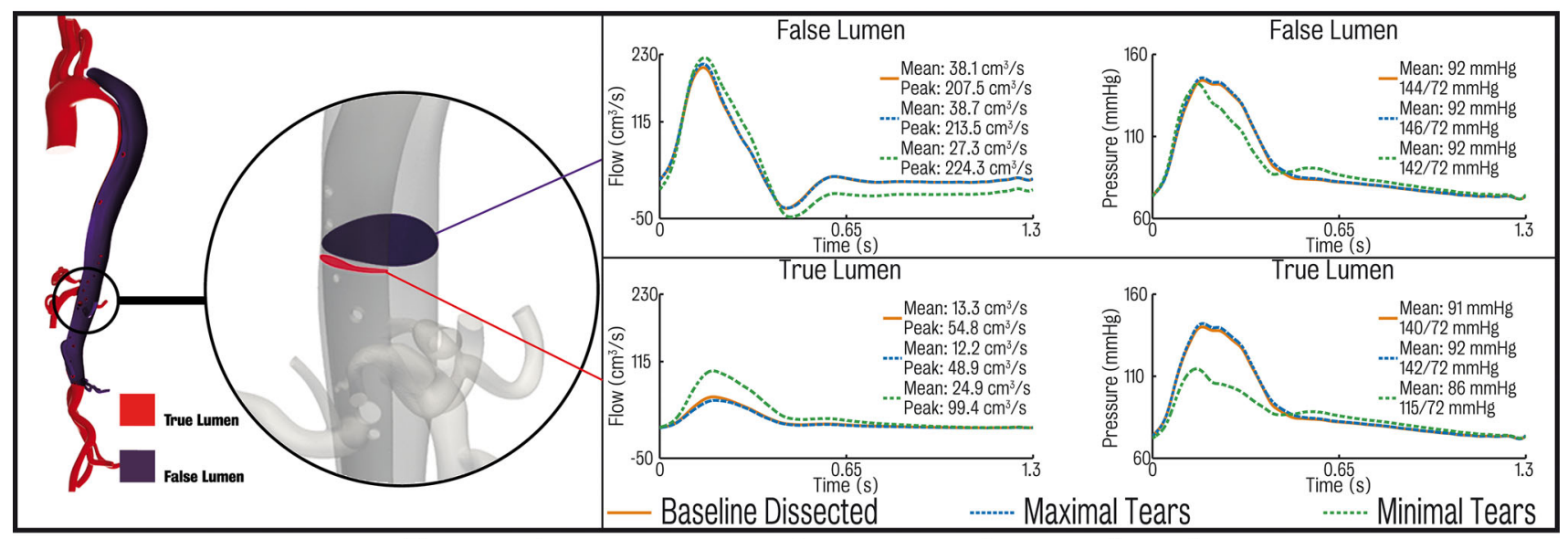

Flow and pressure waveforms at celiac trunk region

Fig. 12 Top comparison of computed mean flows and 2D PC-MRI data for the proximal, medial locations of the descending thoracic aorta. The table gives average values of error over the three locations. Bottom

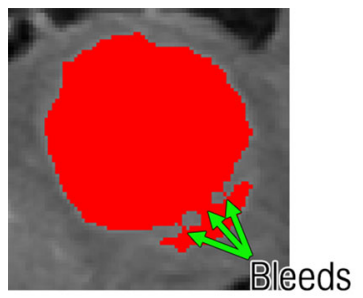

Direct-3D Segmentation

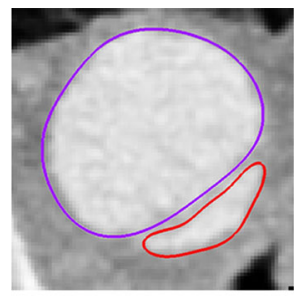

2D Segmentation
Fig. 13 Direct 3D segmentation (left) illustrating typical "bleeds" between the true and false lumina through the relatively thin and poorly defined septum. For comparison, a typical 2D segmentation in the same plane is shown (right) where contours may be automatically created and corrected manually if necessary

In the first study, a comparison between haemodynamics in a subject-specific aortic dissection model and a hypothetical baseline normal (undissected) aorta was performed. The computed pressure and flow waveforms for each of the three dissection models at a location just proximal to the coeliac trunk

undissected model was created by virtually removing the intimal flap of the dissected aorta, assuming that no remodelling (e.g. false lumen expansion) had occurred due to the acute nature of the dissection. Assuming identical cardiac output for both the dissected and undissected models, our results show significant differences between the models and give an estimation of the haemodynamic changes that occurred for this patient with the onset of the dissection. There is an increase in the ascending aortic pulse pressure in the dissected model $(159 / 71 \mathrm{mmHg}$, pulse $88 \mathrm{mmHg})$ compared to the undissected model (146/74 $\mathrm{mmHg}$, pulse $72 \mathrm{mmHg}$ ). These differences are consistent with the larger afterload induced by the dissection septum compared to the baseline model. In the dissected case, the true lumen exhibits a much larger pressure gradient in the descending aorta when compared to the undissected model, particularly in the region proximal to the coeliac trunk. This larger pressure gradient is associated with higher peak velocities and larger wall shear stress. The section distal to the coeliac trunk region shows 
a drop in both the true lumen pressure gradient, mean pressure, and reduced peak velocity and wall shear stress. Local regions of high velocities and wall shear stress are observed where flow through connecting tears impinge on the true lumen wall. Flow through the main entry tear creates a jet that impinges on the false lumen wall, creating a region of highly complex flow, increased pressure and WSS. As Tolenaar et al. (2013a) hypnotised, this region of high pressure in the false lumen may indicate an area of concern as a region where aortic growth is probable. The false lumen carries a greater proportion of descending aortic flow and is significantly larger than the true lumen. The false lumen exhibits a more homogenous pressure gradient along its length, with lower velocities and lower wall shear stress than the true lumen.

In a second study, we investigated the changes in left ventricular workload in aortic dissection. This was done by using a 0D lumped-parameter heart model coupled to the image-based aortic model. Using this approach, left ventricular function can be obtained from the interactions between the heart model and the afterload provided by the aorta and the Windkessels. Left ventricular volume and pressure can be recorded, and a PV loop obtained. The intimal flap and more complex flow patterns in the aorta result in higher energy losses and an effective increase in the hydraulic resistance felt by the heart. The heart model was tuned using values derived from Lau and Figueroa (2015) to match the acquired 2D PC-MRI flow data and then adjusted to produce the same cardiac output in the undissected case. Our results showed that there is an increase in left ventricular stroke work of $13.7 \%$ in the dissected case compared to the undissected case for the same cardiac output. This increase is equivalent to adding a full day of work per week. This is therefore an important consideration to observe in the management of chronic dissection patients, where conservative medical management is often adopted as a long-term strategy.

Previous aortic dissection CFD studies have typically used models consisting of a single entry and/or exit tear (Karmonik et al. 2008; Rudenick et al. 2010; Karmonik et al. 2011b, a; Heim et al. 2013). The effect of varying size and location of entry and exit tears in dissection has been studied (Tsai et al. 2008; Karmonik et al. 2011a; Rudenick et al. 2013), and observational studies have examined the correlation of the number of communication tears and rate of growth of dissection (Tolenaar et al. 2013b). Generally, the relatively small secondary tears have not been included in CFD studies of dissection. For the third study, we have tried, for the first time, to quantify the effect of secondary tears and found that these tears play a significant role in dissection haemodynamics. In our study, while the size of the individual tears was not altered, the number of connecting tears in both the proximal and distal parts of the dissection was. In other words, the total cross-sectional area available for communication between lumina changes as a result of the increase in the number of communicating tears. Our results showed that multiple tears along the length of the dissection result in an equalisation of pressure between the lumina. An increase in false lumen flow was observed when more communicating tears are present. This would seem to be consistent with other clinical and experimental studies which have suggested that these secondary tears have a significant impact on the haemodynamics. Tolenaar et al. (2013b) showed that patients with a single entry tear had significantly poorer outcomes than those with multiple tears while Rudenick et al. (2013) showed that small connecting tears resulted in a larger pressure difference between the lumina with higher pressures observed in the true lumen, while larger tears resulted in an equalisation of pressure between the lumina. The study also showed that the true lumen receives a larger proportion of flow in the case of smaller tears. Berguer et al. (2014) showed that the pressure differential between the true and false lumina is inversely proportional to the cross-sectional area of the exit tear. From the maximal tears results, it would also appear the inclusion of the two tears in the thoracic aorta had little effect on the pressures or flows in the lumina relative to the baseline case, and it may therefore not be necessary to include all connecting tears to ensure relatively accurate modelling of the dissection haemodynamics.

We have also shown that the number of connecting tears results in significant changes in both pressure and flow particularly in the true lumen. This may be due to the high flow demand of the branch vessels around the coeliac trunk: secondary tears may facilitate the required flow to satisfy this demand. In the absence of secondary tears, this demand must be satisfied by the true lumen entirely. It is however clear that these tears are small in size and therefore difficult to identify with current imaging technology.

\subsection{Limitations and future work}

\subsubsection{Imaging limitations}

The primary imaging modality used to create the aortic dissection computer model was non-gated contrast-enhanced CT. It has been observed that non-gated CT may contain pulsation artefacts that can result in the generation of an artificial intimal flap (Qanadli et al. 1999; Flohr et al. 2002). There are inherent disadvantages to gated CT such as higher radiation dose and longer scan time. However, gating does allow for the capture of the septum at the same phase in the cardiac cycle, thereby eliminating motion due to pulsation. Imaging the septum in such a way should eliminate the majority of motion artefacts and allow the reconstruction of a more accurate representation of the geometry.

It is generally desirable to have higher spatial resolution for both CT and MRI data when performing image segmen- 

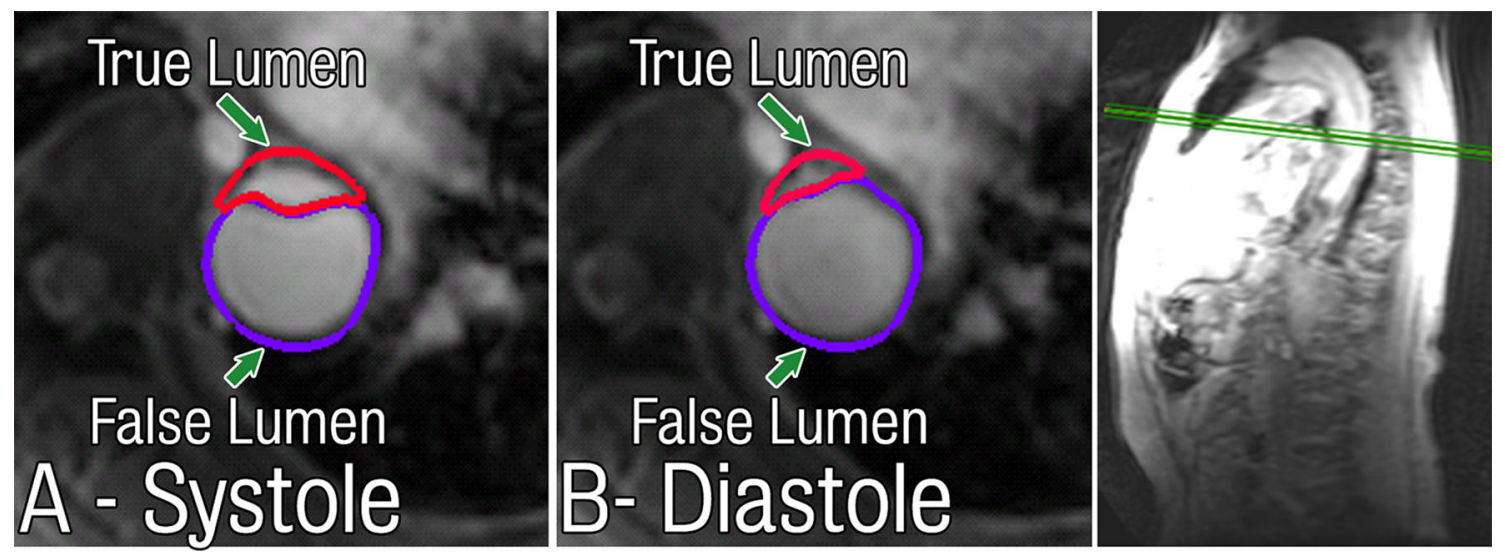

Fig. 14 2D MRI showing distention of the dissection flap between peek systole (A) and mid-diastole (B)

tation as low spatial resolution limits the size of features that may be included in the computer model, and, in this instance, made identification of the relatively small secondary tears difficult. Twenty-five images were acquired for both 2D and 4D PC-MRI data sets resulting in a temporal resolution of $52 \mathrm{~ms}$. When acquiring PC-MRI data, higher temporal resolution may enable better observation of the septum motion. Higher temporal and spatial resolution of 4D PC-MRI should allow for flow patterns through tears to be more readily observed.

The 2D PC-MRI data were acquired at three locations in close proximity in the thoracic region of the aorta. A greater number of 2D PC-MRI scans capturing flow in the coeliac and iliac regions may have enabled a better, more accurate tuning of the outflow Windkessel models. Furthermore, dual VENC (velocity encoding) in the descending aorta would have increased the fidelity of the reconstructed true lumen and false lumen flows since significantly different maximum velocities are found in the two lumina.

The results indicate that inclusion of the difficult to image connecting tears is necessary for accurate modelling. Arterial spin labelling (ASL) is an MRI technique which allows the tracking of labelled blood over time (Wong 2014). This technique could be used in dissection patients to identify secondary connecting tears by detecting the transfer of labelled blood between the lumina. Lastly, initial results using blackblood imaging techniques have yielded excellent results in imaging the intimal flap accurately. This technique avoids the exposure to radiation of $\mathrm{CT}$ we hope to use this technique for image acquisition in cases of aortic dissection.

\subsubsection{Pressure data}

In this study, we aimed to reproduce physiologic values of pressure in the thoracic aorta since we did not have subjectspecific pressure data. This is an important limitation as the pressure in true and false lumina may differ signifi- cantly depending of the size and number of connecting tears (Rudenick et al. 2013). In our paper, the large size of the connecting tears $(13 \mathrm{~mm}$ proximally and $9.3 \mathrm{~mm}$ distally) makes the differences between true and false lumina pressure modest, and therefore, the lack of pressure data may be less critical. In future studies, patient-specific pressure data using soft, pliable wires with a high-fidelity sensors will be collected at multiple locations in the aorta from patients during planned endovascular procedures. These pressure data will provide a gold standard for the simulated CFD pressures.

\subsubsection{Modelling limitations}

The main limitation in the modelling approach of this paper is the rigid wall assumption. Imaging of the intimal flap using 2D MRI techniques shows portions of the flap that undergo significant deformations during systole (see Fig. 14). Other studies have shown that the true lumen experiences expansion and forward flow during systole, and an almost complete collapse during diastole due to pressure fluctuations. This may increase the risk of end-organ ischaemia. False lumen flow can be seen to be delayed, absent or even reversed, depending on the geometry configuration of the tear and the degree of communication between the true and false lumina (Erbel et al. 2001).

The mismatch in amplitude between the computed and measured flow wave forms observed is due to the lack of compliance in both the aortic wall and intimal flap of the computer model. This compliance can only be represented via a suitable fluid-structure interaction (FSI) method. A FSI method will however increase the computational cost of the simulations significantly. In recent years, non-boundary- fitted methods pioneered by Peskin (1972) have been developed to represent the dynamics of thin structures embedded in a fluid. These methods, such as the Fictitious domain method (Glowinski et al. 1994), the mortar element method (Baai- 
jens 2001) and the immersed structural potential method (ISPM) (Gil et al. 2010) allow for the imposition of the necessary kinematic (no-slip) and dynamic (balance of forces) compatibility conditions without resorting to computationally more expensive methods that require continuous update of the computational grid as in boundary-fitted Arbitrary Lagrangian Eulerian (ALE) formulations (Nordsletten et al. 2010a,b).

When comparing haemodynamics in the dissected and undissected models, two main assumptions were made. First, given the acute nature of the dissection, we assumed that no false lumen remodelling had taken place, and therefore, we simply removed the intimal flap in the dissection model to create a model of the undissected geometry. This was justified by the acute nature of the aortic dissection. In acute dissection, there is minimal remodelling of the lumina and septum. Although the false lumen can potentially enlarge acutely due to its smaller thickness (Masuda et al. 1992; Fisher et al. 1994; Erbel et al. 2001; Khan and Nair 2002), this expansion was not noted in this study. Second, we neglected the potential responses of both the baroreflex and the cerebral auto-regulation (Lau and Figueroa 2015), which are likely to react to changes in aortic pressure and upper branch flow following aortic dissection. Ignoring the baroreflex and local auto-regulations resulted in assuming identical inflow and outflow boundary conditions for both the dissected and undissected cases.

Since no flow data were available prior to the onset of the dissection, it was assumed that the cardiac output remained unchanged pre- and post-dissection. This assumption implies that the heart adapted to changes in afterload with the overall objective to maintain the cardiac output constant.

The heart model allows the estimation of stroke work, but as there was no image data available on ventricular volumes, a number of assumptions were made for the heart lumpedparameter model such as end diastolic volume and preload. Our heart parameter models were taken from a previously published study (Lau and Figueroa 2015). As such, the stroke work shown is an estimation of the cardiac workload. Future studies may benefit from the acquisition of cine image data of the heart.

\section{Conclusions}

In this study, multi-modality image data were used to create and validate a detailed computational model of haemodynamics in the human dissected aorta. This is the first study in which numerical predictions are quantitatively compared against PC-MRI data in the descending aorta. Our results also show that secondary communicating tears, particularly larger tears, have a significant impact on haemodynamics in the descending and thoracic aorta.
There is a clear need for better imaging and computational methods to produce more accurate anatomical and physiological models of aortic dissection. Enhanced image-based simulation methods hold the key to improving our current understanding of the biomechanics of this complex disease. This better understanding will make it possible to devise better strategies for the long-term management of patients with chronic dissection, allowing for the identification of potential areas of aortic growth, ensuring visceral organs are adequately perfused and potentially aiding in surgical planning.

Acknowledgments The authors acknowledge support from the European Research Council under the European Union's Seventh Framework Programme (FP/2007-2013) / ERC Grant Agreement No. 307532, Royal College of Surgeons of England (RG I13142461), Heart Research UK (RG2629/13/14), BHF New Horizons program (NH/11/5/29058), and the United Kingdom Department of Health via the National Institute for Health Research (NIHR) comprehensive Biomedical Research Centre award to Guy's and St Thomas' NHS Foundation Trust in partnership with King's College London and King's College Hospital NHS Foundation Trust. The authors also wish to acknowledge Simmetrix, Inc. (http://www.simmetrix.com/) for their MeshSim mesh generation library. Finally, thanks and acknowledgements to Drs. Rostislav Khlebnikov, Chris Arthurs, Kevin Lau and Nan Xiao for their assistance and feedback.

Open Access This article is distributed under the terms of the Creative Commons Attribution 4.0 International License (http://creativecomm ons.org/licenses/by/4.0/), which permits unrestricted use, distribution, and reproduction in any medium, provided you give appropriate credit to the original author(s) and the source, provide a link to the Creative Commons license, and indicate if changes were made.

\section{References}

Alastruey J, Parker KH, Peiró J et al (2007) Modelling the circle of Willis to assess the effects of anatomical variations and occlusions on cerebral flows. J Biomech 40:1794-1805. doi:10.1016/j.jbiomech. 2006.07.008

Alimohammadi M, Agu O, Balabani S, Díaz-Zuccarini V (2014) Development of a patient-specific simulation tool to analyse aortic dissections: assessment of mixed patient-specific flow and pressure boundary conditions. Med Eng Phys 36:275-284. doi:10.1016/j. medengphy.2013.11.003

Baaijens FPT (2001) A fictitious domain/mortar element method for fluid-structure interaction. Int $\mathrm{J}$ Numer Methods Fluids 35:743-761. doi:10.1002/1097-0363(20010415)35:7<743:: AID-FLD109>3.0.CO;2-A

Berguer R, Parodi JC, Schlicht M, Khanafer K (2014) Experimental and clinical evidence supporting septectomy in the primary treatment of acute type B thoracic aortic dissection. Ann Vasc Surg 29:167173. doi:10.1016/j.avsg.2014.10.001

Bernstein MA, Zhou XJ, Polzin JA et al (1998) Concomitant gradient terms in phase contrast MR: analysis and correction. Magn Reson Med 39:300-308. doi:10.1002/mrm. 1910390218

Chen D, Müller-Eschner M, Kotelis D et al (2013) A longitudinal study of Type-B aortic dissection and endovascular repair scenarios: computational analyses. Med Eng Phys 35:1321-1330. doi:10. 1016/j.medengphy.2013.02.006 
Cheng Z, Tan FPP, Riga CV et al (2010) Analysis of flow patterns in a patient-specific aortic dissection model. J Biomech Eng 132:051007. doi:10.1115/1.4000964

CIBC (2015) Imaging Institute (SCI). Seg3d: volumetric image segmentation and visualization. www.seg3d.org

Clough RE, Waltham M, Giese D et al (2012) A new imaging method for assessment of aortic dissection using four-dimensional phase contrast magnetic resonance imaging. J Vasc Surg 55:914-923. doi:10.1016/j.jvs.2011.11.005

Coady M, Ikonomidis JS, Cheung AT et al (2010) Surgical management of descending thoracic aortic disease: open and endovascular approaches a scientific statement from the American Heart Association. Circulation 121:2780-2804. doi:10.1161/CIR. 0b013e3181e4d033

CRIMSON (2015). www.crimson.software

Elefteriades JA, Lovoulos CJ, Coady MA et al (1999) Management of descending aortic dissection. Ann Thorac Surg 67:2002-2005. doi:10.1016/S0003-4975(99)00428-2

Erbel R, Alfonso F, Boileau C et al (2001) Diagnosis and management of aortic dissection. Eur Heart J 22:1642-1681. doi:10.1053/euhj. 2001.2782

Fisher ER, Stern EJ, Godwin JD 2nd et al (1994) Acute aortic dissection: typical and atypical imaging features. Radiographics 14:12631271. doi:10.1148/radiographics.14.6.7855340

Flohr T, Prokop M, Becker C et al (2002) A retrospectively ECGgated multislice spiral CT scan and reconstruction technique with suppression of heart pulsation artifacts for cardio-thoracic imaging with extended volume coverage. Eur Radiol 12:1497-1503. doi:10.1007/s00330-002-1388-0

Formaggia L, Lamponi D, Tuveri M, Veneziani A (2006) Numerical modeling of $1 \mathrm{D}$ arterial networks coupled with a lumped parameters description of the heart. Comput Methods Biomech Biomed Eng 9:273-288. doi:10.1080/10255840600857767

Formaggia L, Nobile F, Quarteroni A, Veneziani A (1999) Multiscale modelling of the circulatory system: a preliminary analysis. Comput Vis Sci 2:75-83. doi:10.1007/s007910050030

Gatehouse PD, Rolf MP, Graves MJ et al (2010) Flow measurement by cardiovascular magnetic resonance: a multi-centre multi-vendor study of background phase offset errors that can compromise the accuracy of derived regurgitant or shunt flow measurements. J Cardiovasc Magn Reson 12:5. doi:10.1186/1532-429X-12-5

Gil AJ, Arranz Carreño A, Bonet J, Hassan O (2010) The immersed structural potential method for haemodynamic applications. J Comput Phys 229:8613-8641. doi:10.1016/j.jcp.2010.08.005

Glowinski R, Pan TW, Periaux J (1994) A fictitious domain method for Dirichlet problem and applications. Comput Methods Appl Mech Eng 111:283-303. doi:10.1016/0045-7825(94)90135-X

Heim L, Poole RJ, Warwick R, Poullis M (2013) The concept of aortic replacement based on computational fluid dynamic analysis: patient-directed aortic replacement. Interact Cardiovasc Thorac Surg 16:583-587. doi:10.1093/icvts/ivt031

Hughes TJR, Wells GN (2005) Conservation properties for the Galerkin and stabilised forms of the advection-diffusion and incompressible Navier-Stokes equations. Comput Methods Appl Mech Eng 194:1141-1159. doi:10.1016/j.cma.2004.06.034

Hunter PJ, Pullan AJ, Smaill BH (2003) Modeling total heart function. Annu Rev Biomed Eng 5:147-177. doi:10.1146/annurev.bioeng. 5.040202.121537

Jansen KE, Whiting CH, Hulbert GM (2000) A generalized- $\alpha$ method for integrating the filtered Navier-Stokes equations with a stabilized finite element method. Comput Methods Appl Mech Eng 190:305-319. doi:10.1016/S0045-7825(00)00203-6

Karmonik C, Bismuth J, Redel T et al (2010) Impact of tear location on hemodynamics in a type $\mathrm{B}$ aortic dissection investigated with computational fluid dynamics. Conf Proc IEEE Eng Med Biol Soc 2010:3138-3141. doi:10.1109/IEMBS.2010.5627193
Karmonik C, Bismuth J, Shah DJ et al (2011) Computational study of haemodynamic effects of entry-and exit-tear coverage in a DeBakey type III aortic dissection: technical report. Eur J Vasc Endovasc Surg 42:172-177. doi:10.1016/j.ejvs.2011.04.008

Karmonik C, Bismuth J, Shah DJ et al (2011b) A computational fluid dynamics study pre- and post-stent graft placement in an acute type B aortic dissection. Vasc Endovasc Surg 45:157-164. doi:10. $1177 / 1538574410389342$

Karmonik C, Bismuth JX, Davies MG, Lumsden AB (2008) Computational hemodynamics in the human aorta: a computational fluid dynamics study of three cases with patient-specific geometries and inflow rates. Technol Heal Care 16:343-354

Karmonik C, Muller-Eschner M, Partovi S et al (2013) Computational fluid dynamics investigation of chronic aortic dissection hemodynamics versus normal aorta. Vasc Endovasc Surg 47:625-631. doi: $10.1177 / 1538574413503561$

Karmonik C, Partovi S, Müller-Eschner M et al (2012) Longitudinal computational fluid dynamics study of aneurysmal dilatation in a chronic DeBakey type III aortic dissection. J Vasc Surg 56:2603.e1. doi:10.1016/j.jvs.2012.02.064

Kerckhoffs RCP, Neal ML, Gu Q et al (2006) Coupling of a 3D finite element model of cardiac ventricular mechanics to lumped systems models of the systemic and pulmonic circulation. Ann Biomed Eng 35:1-18. doi:10.1007/s10439-006-9212-7

Khan IA, Nair CK (2002) Clinical, diagnostic, and management perspectives of aortic dissection. CHEST J 122:311-328. doi:10. 1378/chest.122.1.311

Khoynezhad A, Walot I, Kruse MJ et al (2010) Distribution of intimomedial tears in patients with type B aortic dissection. J Vasc Surg 52:562-568. doi:10.1016/j.jvs.2010.04.036

Kim HJ, Vignon-Clementel IE, Figueroa CA et al (2009) On coupling a lumped parameter heart model and a three-dimensional finite element aorta model. Ann Biomed Eng 37:2153-2169. doi:10. 1007/s10439-009-9760-8

Lau KD, Figueroa CA (2015) Simulation of short-term pressure regulation during the tilt test in a coupled 3D-0D closed-loop model of the circulation. Biomech Model Mechanobiol 14:915-929. doi:10. 1007/s10237-014-0645-x

Markl M, Bammer R, Alley MT et al (2003) Generalized reconstruction of phase contrast MRI: analysis and correction of the effect of gradient field distortions. Magn Reson Med 50:791-801. doi:10. 1002/mrm. 10582

Masuda Y, Takanashi K, Takasu J et al (1992) Expansion rate of thoracic aortic aneurysms and influencing factors. Chest 102:461-466. doi:10.1378/chest.102.2.461

McGee EC, Pham DT, Gleason TG (2005) Chronic descending aortic dissections. Semin Thorac Cardiovasc Surg 17:262-267. doi:10. 1053/j.semtcvs.2005.06.013

McMahon MA, Squirrell CA (2010) Multidetector CT of aortic dissection: a pictorial review. Radiographics 30:445-460. doi:10.1148/ rg.302095104

Nienaber CA, Eagle KA (2003) Aortic dissection: new frontiers in diagnosis and management Part II-therapeutic management and follow-up. Circulation 108:772-778. doi:10.1161/01.CIR. 0000087400.48663.19

Nordsletten D, Kay D, Smith N (2010a) A non-conforming monolithic finite element method for problems of coupled mechanics. J Comput Phys 229:7571-7593. doi:10.1016/j.jcp.2010.05.043

Nordsletten D, Mccormick M, Kilner PJ et al (2010b) Fluid-solid coupling for the investigation of diastolic and systolic human left ventricular function. Int J Numer Method Biomed Eng 27:10171039. doi: $10.1002 / \mathrm{cnm} .1405$

Ottesen JT, Olufsen MS, Larsen JK (2004) Applied mathematical models in human physiology. Society for Industrial and Applied Mathematics, Philadelphia 
Peskin C (1972) Flow patterns around heart valves: a numerical method. J Comput Phys 271:252-271. doi:10.1016/ 0021-9991(72)90065-4

Pope S, Ellwein L, Zapata C et al (2009) Estimation and identification of parameters in a lumped cerebrovascular model. Math Biosci Eng 6:93-115. doi:10.3934/mbe.2009.6.93

Qanadli SD, El Hajjam M, Mesurolle B et al (1999) Motion artifacts of the aorta simulating aortic dissection on spiral CT. J Comput Assist Tomogr 23:1-6. doi:10.1097/00004728-199901000-00001

Quint LE, Platt JF, Sonnad SS et al (2003) Aortic intimal tears: detection with spiral computed tomography. J Inf 10:505-510. doi:10.1583/ 1545-1550(2003)010<0505:AITDWS > 2.0.CO;2

Rudenick PA, Bijnens BH, García-Dorado D, Evangelista A (2013) An in vitro phantom study on the influence of tear size and configuration on the hemodynamics of the lumina in chronic type B aortic dissections. J Vasc Surg 57:464-474. doi:10.1016/j.jvs.2012.07. 008

Rudenick PA, Bordone M, Bijnens BH et al (2010) A multi-method approach towards understanding the pathophysiology of aortic dissections - the complementary role of in-silico, in-vitro and invivo information. In: Statistical atlases and computational models of the heart. Berlin, pp 114-123

Sahni O, Müller J, Jansen KE et al (2006) Efficient anisotropic adaptive discretization of the cardiovascular system. Comput Methods Appl Mech Eng 195:5634-5655. doi:10.1016/j.cma.2005.10.018

Shahcheraghi N, Dwyer HA, Cheer AY et al (2002) Unsteady and threedimensional simulation of blood flow in the human aortic arch. J Biomech Eng 124:378. doi:10.1115/1.1487357

Stergiopulos N, Young DF, Rogge TR (1992) Computer simulation of arterial flow with applications to arterial and aortic stenoses. J Biomech 25:1477-1488. doi:10.1016/0021-9290(92)90060-E

Takami Y, Tajima K, Kato W et al (2012) Can we predict the site of entry tear by computed tomography in patients with acute type A aortic dissection? Clin Cardiol 35:500-504. doi:10.1002/clc.21991

Tam ASM, Catherine Sapp M, Roach MR (1998) The effect of tear depth on the propagation of aortic dissections in isolated porcine thoracic aorta. J Biomech 31:673-676. doi:10.1016/ S0021-9290(98)00058-X

Taylor CA, Figueroa CA (2009) Patient-specific modeling of cardiovascular mechanics. Annu Rev Biomed Eng 11:109-134. doi:10. 1146/annurev.bioeng.10.061807.160521

Tolenaar JL, van Keulen JW, Jonker FHW et al (2013) Morphologic predictors of aortic dilatation in type B aortic dissection. J Vasc Surg 58:1220-1225. doi:10.1016/j.jvs.2013.05.031

Tolenaar JL, van Keulen JW, Trimarchi S et al (2013) Number of entry tears is associated with aortic growth in type B dissections. Ann Thorac Surg 96:39-42. doi:10.1016/j.athoracsur.2013.03.087

Tsai TT, Evangelista A, Nienaber CA et al (2007) Partial thrombosis of the false lumen in patients with acute type B aortic dissection. $\mathrm{N}$ Engl J Med 357:349-359. doi:10.1056/NEJMoa063232
Tsai TT, Schlicht MS, Khanafer K et al (2008) Tear size and location impacts false lumen pressure in an ex vivo model of chronic type $\mathrm{B}$ aortic dissection. J Vasc Surg 47:844-851. doi:10.1016/j.jvs.2007. 11.059

Tse KM, Chiu P, Lee HP, Ho P (2011) Investigation of hemodynamics in the development of dissecting aneurysm within patient-specific dissecting aneurismal aortas using computational fluid dynamics (CFD) simulations. J Biomech 44:827-836. doi:10.1016/j. jbiomech.2010.12.014

Vignon-Clementel IE, Figueroa CA, Jansen KE, Taylor CA (2010) Outflow boundary conditions for 3D simulations of non-periodic blood flow and pressure fields in deformable arteries. Comput Methods Biomech Biomed Eng 13:625-640. doi:10.1080/ 10255840903413565

Vignon-Clementel IE, Figueroa CA, Jansen KE, Taylor CA (2006) Outflow boundary conditions for three-dimensional finite element modeling of blood flow and pressure in arteries. Comput Methods Appl Mech Eng 195:3776-3796. doi:10.1016/j.cma.2005.04.014

Wang KC, Dutton RW, Taylor CA (1999) Improving geometric model construction for blood flow modeling: geometric image segmentation and image-based model construction for computational hemodynamics. IEEE Eng Med Biol Mag 18:33-39. doi:10.1109/ 51.805142

Westerhof N, Lankhaar J-W, Westerhof BE (2009) The arterial Windkessel. Med Biol Eng Comput 47:131-141. doi:10.1007/ s11517-008-0359-2

Whiting CH, Jansen KE (2001) A stabilized finite element method for the incompressible Navier-Stokes equations using a hierarchical basis. Int J Numer Methods Fluids 35:93-116. doi:10.1002/ 1097-0363(20010115)35:1<93::AID-FLD85>3.0.CO;2-G

Willoteaux S, Lions C, Gaxotte V et al (2004) Imaging of aortic dissection by helical computed tomography (CT). Eur Radiol 14:1999-2008. doi:10.1007/s00330-004-2441-y

Wolf I, Vetter M, Wegner I et al (2005) The medical imaging interaction toolkit. Med Image Anal 9:594-604. doi:10.1016/j.media.2005. 04.005

Wong EC (2014) An introduction to ASL labeling techniques. J Magn Reson Imaging. doi:10.1002/jmri.24565

Xiao N, Alastruey J, Figueroa CA (2014) A systematic comparison between 1-D and 3-D hemodynamics in compliant arterial models. Int J Numer Method Biomed Eng 30:204-231. doi:10.1002/cnm. 2598

Xiao N, Humphrey JD, Figueroa CA (2013) Multi-scale computational model of three-dimensional hemodynamics within a deformable full-body arterial network. J Comput Phys 244:22-40. doi:10. 1016/j.jcp.2012.09.016

Yushkevich PA, Piven J, Hazlett HC et al (2006) User-guided 3D active contour segmentation of anatomical structures: significantly improved efficiency and reliability. Neuroimage 31:1116-1128. doi:10.1016/j.neuroimage.2006.01.015 